\title{
Sphere-valued harmonic maps with surface energy and the $K_{13}$ problem
}

\author{
Stuart Day*and Arghir Dani Zarnescu ${ }^{\dagger \ddagger}$
}

October 19, 2017

\begin{abstract}
We consider an energy functional motivated by the celebrated $K_{13}$ problem in the Oseen-Frank theory of nematic liquid crystals. It is defined for spherevalued functions and appears as the usual Dirichlet energy with an additional surface term.

It is known that this energy is unbounded from below and our aim has been to study the local minimizers. We show that even having a critical point in a suitable energy space imposes severe restrictions on the boundary conditions. Having suitable boundary conditions makes the energy functional bounded and in this case we study the partial regularity of the global minimizers.
\end{abstract}

\section{Introduction}

In this paper we study critical points of the following energy functional

$$
\mathbb{E}[n]=\int_{\Omega} \frac{K}{2}|\nabla n|^{2} d x+\tilde{K}_{13} \int_{\partial \Omega}((n \cdot \nabla) n) \cdot \nu d \sigma
$$

for maps $n: \Omega \subset \mathbb{R}^{d} \rightarrow \mathbb{R}^{d}$ with $|n(x)|=1$ a.e. in $\bar{\Omega}$ where

$$
K>0, \tilde{K}_{13} \in \mathbb{R}
$$

*Department of Mathematics, University of Sussex, Pevensey III, Falmer, BN1 9QH, UK Email: S.Day@sussex.ac.uk

${ }^{\dagger}$ Ikerbasque, Basque Foundation for Science, Maria Diaz de Haro 3, 48013, Bilbao, Bizkaia, Spain

$\ddagger$ BCAM, Basque Center for Applied Mathematics, Mazarredo 14, 48009 Bilbao, Bizkaia, Spain Email: azarnescu@bcamath.org

$\S$ "Simion Stoilow" Institute of Mathematics of the Romanian Academy, 21 Calea Griviţei Street, 010702 Bucharest, Romania 
and $\Omega \subset \mathbb{R}^{d}$ with $d \in\{2,3\}$ is a $C^{2}$ domain and $\nu$ denotes the exterior unit-normal.

This functional is motivated by the $K_{13}$ problem in the Oseen-Frank theory of liquid crystals. More details about the physical relevance of this problem are provided in the next subsection, Section 1.1.

It has been known since 1985 thanks to the work of C. Oldano and G. Barbero [14] that there exists a domain $\Omega \subset \mathbb{R}^{3}$ and a sequence $\left\{n_{k}\right\}_{k \in \mathbb{N}} \subset C^{\infty}\left(\Omega ; \mathbb{S}^{2}\right)$ such that $\mathbb{E}\left[n_{k}\right] \rightarrow-\infty$ as $k \rightarrow \infty$. Thus one cannot understand the physical meaning of the energy in the standard way, i.e. from the point of view of global energy minimizers. However it is conceivable that the energy might still have nontrivial local energyminimizers and this has been the starting point of this work.

A first question is then to understand what is the space in which to look for local minimizers. In order to understand this one can start by noting that for $n \in$ $C^{2}\left(\Omega, \mathbb{S}^{d-1}\right)$ an integration by parts allows to rewrite the energy as:

$$
\mathbb{E}[n]=\int_{\Omega} \frac{K}{2}|\nabla n|^{2} d x+\tilde{K}_{13} \int_{\Omega} \sum_{\alpha, \beta=1}^{d}\left(\frac{\partial n_{\alpha}}{\partial x_{\beta}} \frac{\partial n_{\beta}}{\partial x_{\alpha}}+n_{\beta} \frac{\partial^{2} n_{\alpha}}{\partial x_{\alpha} \partial x_{\beta}}\right) d x
$$

A minimal requirement for the functional space is that the energy makes sense for functions in it. Thus a natural choice is:

$$
\mathscr{A}:=W^{1,2}\left(\Omega ; \mathbb{S}^{d-1}\right) \cap W^{2,1}\left(\Omega ; \mathbb{S}^{d-1}\right)
$$

In this space one can consider various boundary conditions, which would make the space smaller. However we surprisingly have that there are severe constraints on what the boundary conditions could be:

THEOREM 1.1. Let $\Omega \subset \mathbb{R}^{d}, d=2,3$ be an open bounded set with $C^{1}$ boundary and with unit-norm exterior normal denoted $\nu$. Consider the energy $\mathbb{E}$ as defined in (1.1).

Let $\bar{n}$ be a critical point of $\mathbb{E}$ in the functional space $\mathscr{A}$ defined in (1.2). Then we have

$$
\bar{n}(x) \cdot \nu(x) \in\{0, \pm 1\}, \text { for almost all } x \in \partial \Omega
$$

The most interesting case is the one when $\bar{n}(x) \cdot \nu(x)=0$ for almost all $x \in \partial \Omega$ and in dimension $d=3$ as this allows for a certain level of freedom at the boundary. Then one can show that the energy $\mathbb{E}$ reduces to:

$$
\mathbb{G}[n]:=\int_{\Omega} K \sum_{\alpha, \beta=1}^{3} \frac{\partial n_{\alpha}}{\partial x_{\beta}} \frac{\partial n_{\alpha}}{\partial x_{\beta}}-\tilde{K}_{13} \int_{\partial \Omega} \sum_{\alpha, \beta=1}^{3} \frac{\partial \nu_{\beta}}{\partial x_{\alpha}} n_{\beta} n_{\alpha} .
$$

This energy makes sense in the large functional space $W^{1,2}\left(\Omega, \mathbb{S}^{2}\right)$ and it is easily shown to be bounded from below and lower semicontinuous. Thus one can now 
consider minimizing $\mathbb{G}$ over the function space of $W^{1,2}$ functions with tangential boundary conditions:

$$
\mathcal{U}:=\left\{u \in W^{1,2}\left(\Omega, \mathbb{S}^{2}\right): \operatorname{Trace}(u) \in \mathcal{T}\right\}
$$

where

$$
\mathcal{T}:=\left\{\gamma \in H^{\frac{1}{2}}\left(\partial \Omega, \mathbb{S}^{2}\right): \gamma(x) \cdot \nu(x)=0 \text { for almost every } x \in \partial \Omega\right\} .
$$

A first issue to consider is wether or not this functional space is non-empty as the topological constraint might make it empty, as shown by the "Hairy Ball" Theorem. Fortunately in our case the regularity at the boundary is weaker than continuous and we have:

Proposition 1.2. Let $\Omega$ be a bounded $C^{2}$ domain in $\mathbb{R}^{3}$. Then the space $\mathcal{U}$ defined in (1.5) is non-empty.

Standard arguments provide the existence of a global minimizer. In general this minimizer might not be continuous at the boundary for topological reasons (think of the example of the "Hairy Ball" theorem). It is then of interest to look into the matter of partial regularity for the global minimizers of $\mathbb{G}$ in the space $\mathcal{U}$. This is related to the works of $\mathrm{R}$. Hardt and F. Lin in [7] and later that of Scheven [15] who considered partially constrained boundary conditions, though only for the Dirichlet functionals. We look into this through a method combining the two approaches in the works mentioned above and taking into account the effect of the surface energy. We can thus show:

THEOREM 1.3. Let $\Omega \subset \mathbb{R}^{3}$ be a $C^{2}$ domain. Then a global energy minimizer of the energy $\mathbb{G}$ (defined in (1.4)) in the space (1.5) is continuous on $\bar{\Omega} \backslash Z$ where $Z$ is a set of one-dimensional Hausdorff measure equal to zero.

The paper is organized as follows: in the next section physical background is provided, to be followed in Section 2 by the example of Barbero and Oldano showing the unboundedness of the energy functional, and then the proof of Theorem 1.1. In the last part, Section 3, we prove Proposition 1.2 and then Theorem 1.3. The appendices contain a number of technical lemmas and the list of notations.

\subsection{Physical motivation}

Nematic liquid crystals are the simplest yet the most used type of liquid crystals, with wide-ranging applications, particularly in displays. The simplest and most comprehensive model used for describing the stationary patterns is related to the Oseen-Frank energy (see for instance [21]) : 


$$
\begin{aligned}
\mathbb{E}_{O F}[n]=\int_{\Omega} K_{1}|\nabla \cdot n|^{2} & +K_{2}|n \cdot(\nabla \times n)|^{2}+K_{3}|n \times(\nabla \times n)|^{2} d x \\
& +\int_{\Omega}\left(K_{2}+K_{24}\right)\left(\operatorname{tr}(\nabla n)^{2}-(\nabla \cdot n)^{2}\right)+K_{13} \nabla \cdot((\nabla \cdot n) n) d x
\end{aligned}
$$

where the vector $n$ is unit-length. Using the identity, valid for $n \in C^{1}\left(\Omega ; \mathbb{S}^{2}\right)$ :

$$
\operatorname{tr}(\nabla n)^{2}+|n \cdot(\nabla \times n)|^{2}+|n \times(\nabla \times n)|^{2}=|\nabla n|^{2}
$$

we have that for "equal elastic constants" $K_{1}=K_{2}=K_{3}$ the Oseen-Frank energy reduces to

$$
\tilde{\mathbb{E}}_{O F}[n]=\int_{\Omega} K_{2}|\nabla n|^{2}+K_{24}\left(\operatorname{tr}(\nabla n)^{2}-(\nabla \cdot n)^{2}\right)+K_{13} \nabla \cdot((\nabla \cdot n) n) d x
$$

The $K_{24}$ term is a null-Lagrangian as we have (see for instance [21],[1]):

$$
\begin{aligned}
& \int_{\Omega} K_{24}\left(\operatorname{tr}(\nabla n)^{2}-(\nabla \cdot n)^{2}\right) d x=\int_{\Omega} \nabla \cdot((n \cdot \nabla) n-(\nabla \cdot n) n) d x \\
= & \int_{\partial \Omega}[(n \cdot \nabla) n-(\nabla \cdot n) n] \cdot \nu d \sigma=\int_{\partial \Omega} D_{t} n: n \otimes \nu-\operatorname{tr}\left(D_{t} n\right) n \cdot \nu d \sigma
\end{aligned}
$$

where $D_{t} n:=\nabla n-\nu \otimes \frac{\partial n}{\partial \nu}$ is a differential operator that involves only tangential derivatives, thus its value depends only on the boundary conditions.

The $K_{13}$ term is different as it can be expressed as a surface integral, but the surface integral does not depend only on tangential derivatives. However, we can remove the tangential contribution to the $K_{13}$ term as follows:

$$
\begin{aligned}
K_{24}\left(\operatorname{tr}(\nabla n)^{2}-(\nabla \cdot n)^{2}\right)+K_{13} \nabla \cdot((\nabla \cdot n) n) & =\left(K_{24}-K_{13}\right)\left(\operatorname{tr}(\nabla n)^{2}-(\nabla \cdot n)^{2}\right) \\
& +K_{13}\left(\operatorname{tr}(\nabla n)^{2}-(\nabla \cdot n)^{2}\right)+K_{13} \nabla \cdot((\nabla \cdot n) n) \\
& =\tilde{K}_{24}\left(\operatorname{tr}(\nabla n)^{2}-(\nabla \cdot n)^{2}\right)+K_{13} \nabla \cdot((n \cdot \nabla) n)
\end{aligned}
$$

thus the energy $\mathbb{E}$ that we consider in (1.1) contains the essential terms capturing the difficulty of the physical $K_{13}$ problem namely that the energy is unbounded from below. The results we obtain in the next section are relevant to the full physical Oseen-Frank energy, with suitable adaptations. 


\section{Constraints on the boundary conditions}

\subsection{The unboundedness of the energy functional}

We show now, by following the example provided in E.Virga's book [21] and inspired by [14] that the energy $\mathbb{E}$ can become unbounded from below, so no global minimizers can exist. Let $\Omega$ be the domain in $\mathbb{R}^{3}$ given by $\Omega:=\{(x, y, z): x, y \in(0, l), z \in$ $(-d,+d)\}$ and consider the functions $n_{\varepsilon}(x, y, z):=\left(\cos \left(\rho_{\varepsilon}(z)\right), 0, \sin \left(\rho_{\varepsilon}(z)\right)\right.$ with $\rho_{\varepsilon}(z)$ given by:

$$
\rho_{\varepsilon}(z):= \begin{cases}\rho_{0}+\varepsilon-\frac{1}{\varepsilon^{3}}\left(z-d+\varepsilon^{2}\right)^{2} & \text { if } z \in\left[d-\varepsilon^{2}, d\right], \\ \rho_{0}+\varepsilon & \text { if } z \in\left(-d+\varepsilon^{2}, d-\varepsilon^{2}\right), \\ \rho_{0}+\varepsilon-\frac{1}{\varepsilon^{3}}\left(z+d-\varepsilon^{2}\right)^{2} & \text { if } z \in\left[-d,-d+\varepsilon^{2}\right] .\end{cases}
$$

A calculation gives that $n_{\varepsilon} \in \mathscr{A}$ and

$$
\mathbb{E}\left[n_{\varepsilon}\right]:=4 l^{2}\left(\frac{K}{3}-K_{13} \frac{\sin \left(2 \rho_{0}\right)}{2 \varepsilon}\right) .
$$

Therefore if we choose $\rho_{0}$ such that $\sin \left(\rho_{0}\right)>0$ then we get

$$
\mathbb{E}\left[n_{\varepsilon}\right] \rightarrow-\infty
$$

proving that $\mathbb{E}$ is unbounded from below in the function space $\mathscr{A}$ (for this specific domain $\Omega$ ).

\subsection{Critical points}

Theorem 1.1 is proved by studying the first variations of the energy $\mathbb{E}$ in $\mathscr{A}$. First we will prove some lemmas; note that in Lemma 2.1 it is important that we are working with $\bar{n} \in W^{2,1}\left(\Omega, \mathbb{S}^{2}\right)$ (hence $\left.-\Delta \bar{n}-\bar{n}|\nabla \bar{n}|^{2} \in L^{1}(\Omega)\right)$.

Lemma 2.1. Let $\bar{n}$ be a critical point of the energy $\mathbb{E}$ in the function space $\mathscr{A}$. Then $\bar{n}$ satisfies the equation

$$
\sum_{\alpha, \beta, \gamma=1}^{d} \int_{\partial \Omega}\left(\varphi_{\beta, \alpha} \bar{n}_{\alpha}-\varphi_{\gamma, \alpha} \bar{n}_{\gamma} \bar{n}_{\alpha} \bar{n}_{\beta}\right) \nu_{\beta} d S=0
$$

for all $\varphi \in C^{\infty}\left(\bar{\Omega}, \mathbb{R}^{3}\right)$ such that $\varphi=0$ on $\partial \Omega$. 
Proof. Let $\bar{n}$ be a critical point of $\mathbb{E}$ in $\mathscr{A}$. First, let $\psi \in C_{c}^{\infty}\left(\Omega, \mathbb{R}^{3}\right)$ and set $\bar{n}^{\varepsilon}:=$ $\frac{\bar{n}+\varepsilon \psi}{|\bar{n}+\varepsilon \psi|}$, then $\bar{n}_{\alpha}^{\varepsilon}=\bar{n}_{\alpha}+\varepsilon\left(\psi_{\alpha}-\bar{n}_{\alpha} \bar{n}_{\beta} \psi_{\beta}\right)+O\left(\varepsilon^{2}\right)$ as $\varepsilon \rightarrow 0$ and a standard calculation gives

$$
\left.\frac{d}{d \varepsilon} \mathbb{E}\left[\bar{n}^{\varepsilon}\right]\right|_{\varepsilon=0}=K \int_{\Omega} \nabla \bar{n} \cdot \nabla \psi-|\nabla \bar{n}|^{2} n \cdot \psi d x=0
$$

We now use the fact that $\bar{n} \in W^{2,1}\left(\Omega, \mathbb{S}^{2}\right)$ to perform an integration by parts, giving

$$
\left.\frac{d}{d \varepsilon} \mathbb{E}\left[\bar{n}^{\varepsilon}\right]\right|_{\varepsilon=0}=K \int_{\Omega}\left(-\Delta \bar{n}-|\nabla \bar{n}|^{2} \bar{n}\right) \cdot \psi d x=0 .
$$

As $\psi$ can be chosen arbitrarily and $-\Delta \bar{n}-\bar{n}|\nabla \bar{n}|^{2} \in L^{1}(\Omega)$, for variations in the interior of $\Omega$ we obtain the harmonic map equation for maps into spheres: $\Delta \bar{n}+$ $|\nabla \bar{n}|^{2} \bar{n}=0$ holding almost everywhere.

Next, let $\varphi \in C^{\infty}\left(\bar{\Omega}, \mathbb{R}^{3}\right)$ such that $\varphi=0$ on $\partial \Omega$ and set $\bar{n}^{\varepsilon}=\frac{\bar{n}+\varepsilon \varphi}{|\bar{n}+\varepsilon \varphi|}$. Recalling that we have $\bar{n}_{\alpha}^{\varepsilon}=\bar{n}_{\alpha}+\varepsilon\left(\varphi_{\alpha}-\bar{n}_{\alpha} \bar{n}_{\beta} \varphi_{\beta}\right)+O\left(\varepsilon^{2}\right)$ we obtain on the boundary the following:

$$
\begin{aligned}
\left.\frac{d}{d \varepsilon}\right|_{\varepsilon=0} \nu_{\beta}\left(\bar{n}_{\alpha}^{\varepsilon} \partial_{\alpha} \bar{n}_{\beta}^{\varepsilon}\right) & =\nu_{\beta}\left(\left(\varphi_{\alpha}-\bar{n}_{\alpha} \bar{n}_{\beta} \varphi_{\beta}\right) \partial_{\alpha} \bar{n}_{\beta}+\bar{n}_{\alpha} \partial_{\alpha}\left(\varphi_{\beta}-\bar{n}_{\beta} \bar{n}_{\gamma} \varphi_{\gamma}\right)\right) \\
& =\nu_{\beta}\left(\varphi_{\alpha} \partial_{\alpha} \bar{n}_{\beta}+\bar{n}_{\alpha} \partial_{\alpha} \varphi_{\beta}-\bar{n}_{\alpha} \partial_{\alpha}\left(\bar{n}_{\beta} \bar{n}_{\gamma}\right) \varphi_{\gamma}-\bar{n}_{\alpha} \bar{n}_{\beta} \bar{n}_{\gamma} \partial_{\alpha} \varphi_{\gamma}\right) \\
& =\nu_{\beta}\left(+\bar{n}_{\alpha} \partial_{\alpha} \varphi_{\beta}-\bar{n}_{\alpha} \bar{n}_{\beta} \bar{n}_{\gamma} \partial_{\alpha} \varphi_{\gamma}+\varphi_{\alpha}\left(\partial_{\alpha} \bar{n}_{\beta}-\bar{n}_{\sigma} \partial_{\sigma}\left(\bar{n}_{\beta} \bar{n}_{\alpha}\right)\right)\right) \\
& =\nu_{\beta}\left(+\bar{n}_{\alpha} \partial_{\alpha} \varphi_{\beta}-\bar{n}_{\alpha} \bar{n}_{\beta} \bar{n}_{\gamma} \partial_{\alpha} \varphi_{\gamma}\right)
\end{aligned}
$$

where for the second equality we used that $|n|=1$ hence $n_{\beta} \partial_{\alpha} n_{\beta}=0$. For the last equality we used that $\varphi$ is zero on the boundary.

Using the last calculation we get:

$$
\begin{aligned}
\left.\frac{d}{d \varepsilon} \mathbb{E}\left[\bar{n}^{\varepsilon}\right]\right|_{\varepsilon=0} & =K \int_{\Omega} \nabla \bar{n} \cdot \nabla \varphi-|\nabla \bar{n}|^{2} \bar{n} \cdot \varphi d x+K_{13} \sum_{\alpha, \beta, \gamma=1}^{d} \int_{\partial \Omega}\left(\varphi_{\beta, \alpha} \bar{n}_{\alpha}-\varphi_{\gamma, \alpha} \bar{n}_{\gamma} \bar{n}_{\alpha} \bar{n}_{\beta}\right) \nu_{\beta} d S \\
& =K \int_{\Omega}\left(-\Delta \bar{n}-|\nabla \bar{n}|^{2} \bar{n}\right) \cdot \varphi d x+K_{13} \sum_{\alpha, \beta, \gamma=1}^{d} \int_{\partial \Omega}\left(\varphi_{\beta, \alpha} \bar{n}_{\alpha}-\varphi_{\gamma, \alpha} \bar{n}_{\gamma} \bar{n}_{\alpha} \bar{n}_{\beta}\right) \nu_{\beta} d S \\
& =K_{13} \sum_{\alpha, \beta, \gamma=1}^{d} \int_{\partial \Omega}\left(\varphi_{\beta, \alpha} \bar{n}_{\alpha}-\varphi_{\gamma, \alpha} \bar{n}_{\gamma} \bar{n}_{\alpha} \bar{n}_{\beta}\right) \nu_{\beta} d S,
\end{aligned}
$$

which proves the lemma (note that in passing from the first to second line we used that $\varphi=0$ on the boundary, and from the second to third that $-\Delta \bar{n}-\bar{n}|\nabla \bar{n}|^{2}=0$ ). 
We now prove an analogue of the fundamental lemma of the Calculus of Variations.

Lemma 2.2. Let $d \in\{2,3\}, \Omega \subset \mathbb{R}^{d}$ be a $C^{1}$ domain and $g \in L^{\infty}(\partial \Omega, \mathbb{R})$. Suppose that

$$
\int_{\partial \Omega} \frac{\partial \varphi}{\partial \nu} g(x) d S(x)=0
$$

for all $\varphi \in C^{\infty}(\bar{\Omega}, \mathbb{R})$ such that $\left.\varphi\right|_{\partial \Omega}=0$, where $\nu(x)$ is the unit norm to $\partial \Omega$ at $x$. Then

$$
g(x)=0
$$

for almost all points $x \in \partial \Omega$.

Proof. We prove for the case $d=3$, the case $d=2$ is a simpler version. Let $x_{0} \in \partial \Omega$ be an arbitrary Lebesgue point of $g$ and let $\psi:(-\varepsilon, \varepsilon)^{2} \rightarrow \partial \Omega$ be a coordinate patch such that $\psi(0,0)=x_{0}$. If we choose $\varepsilon>0$ sufficiently small then the map

$$
\begin{aligned}
H:[0, \varepsilon) \times(-\varepsilon, \varepsilon) \times(-\varepsilon, \varepsilon) & \rightarrow \Omega \\
(r, s, t) & \rightarrow[\psi(s, t)-r \nu(s, t)]
\end{aligned}
$$

(where $\nu(s, t)=\frac{\psi_{s} \times \psi_{t}}{\left\|\psi_{s} \times \psi_{t}\right\|}$ is the unit normal to $\partial \Omega$ at $\left.\psi(s, t)\right)$ provides a $C^{1}$ homeomorphism from $(0, \varepsilon) \times(-\varepsilon, \varepsilon) \times(-\varepsilon, \varepsilon)$ onto a relative neighbourhood $U:=H([0, \varepsilon) \times$ $(-\varepsilon, \varepsilon) \times(-\varepsilon, \varepsilon))$ of $x_{0}$. For $\delta>0$ small define the maps

$$
\varphi_{\delta}(x)= \begin{cases}0 & \text { if } x \notin U \\ \left(\frac{\varepsilon}{2 \delta}\right)^{2} \gamma\left(r, \frac{s \varepsilon}{2 \delta}, \frac{t \varepsilon}{2 \delta}\right) & \text { if } x \in U, \text { where } x=\psi(s, t)-r \nu(s, t) .\end{cases}
$$

where

$$
\gamma:[0, \varepsilon) \times(-\varepsilon, \varepsilon) \times(-\varepsilon, \varepsilon) \rightarrow \mathbb{R}
$$

is smooth and 0 if $(r, s, t) \notin\left[0, \frac{\varepsilon}{2}\right) \times\left(-\frac{\varepsilon}{2}, \frac{\varepsilon}{2}\right) \times\left(-\frac{\varepsilon}{2}, \frac{\varepsilon}{2}\right)$. Then we have

$$
\int_{\partial \Omega} \frac{\partial \varphi_{\delta}}{\partial \nu} g(x) d x=-\int_{-\delta}^{\delta} \int_{-\delta}^{\delta}\left(\frac{\varepsilon}{2 \delta}\right)^{2} \frac{\partial \gamma\left(r, \frac{s \varepsilon}{2 \delta}, \frac{t \varepsilon}{2 \delta}\right)}{\partial r} g(\psi(s, t))\left\|\psi_{s} \times \psi_{t}\right\| d s d t=0 .
$$

Using the change of variables $\sigma=\frac{s \varepsilon}{2 \delta}$ and $\theta=\frac{t \varepsilon}{2 \delta}$, we get

$$
\int_{-\frac{\varepsilon}{2}}^{\frac{\varepsilon}{2}} \int_{-\frac{\varepsilon}{2}}^{\frac{\varepsilon}{2}} \frac{\partial \gamma(r, \sigma, \theta)}{\partial r} g\left(\psi\left(\frac{2 \delta \sigma}{\varepsilon}, \frac{2 \delta \theta}{\varepsilon}\right)\right)\left\|\psi_{s}\left(\frac{2 \delta \sigma}{\varepsilon}, \frac{2 \delta \theta}{\varepsilon}\right) \times \psi_{t}\left(\frac{2 \delta \sigma}{\varepsilon}, \frac{2 \delta \theta}{\varepsilon}\right)\right\| d \sigma d \theta=0 .
$$

On the other hand we have: 


$$
\begin{aligned}
& \left|\int_{-\frac{\varepsilon}{2}}^{\frac{\varepsilon}{2}} \int_{-\frac{\varepsilon}{2}}^{\frac{\varepsilon}{2}} \frac{\partial \gamma}{\partial r}(0, s, t)\left(g\left(\psi\left(\frac{2 \delta s}{\varepsilon}, \frac{2 \delta t}{\varepsilon}\right)\right)\left\|\psi_{s} \times \psi_{t}\left(\frac{2 \delta s}{\varepsilon}, \frac{2 \delta t}{\varepsilon}\right)\right\|-g(\psi(0,0))\left\|\psi_{s} \times \psi_{t}(0,0)\right\|\right) d s d t\right| \\
& \leq\left|\int_{-\frac{\varepsilon}{2}}^{\frac{\varepsilon}{2}} \int_{-\frac{\varepsilon}{2}}^{\frac{\varepsilon}{2}} \frac{\partial \gamma}{\partial r}(0, s, t)\left\|\psi_{s} \times \psi_{t}\left(\frac{2 \delta s}{\varepsilon}, \frac{2 \delta t}{\varepsilon}\right)\right\|\left(g\left(\psi\left(\frac{2 \delta s}{\varepsilon}, \frac{2 \delta t}{\varepsilon}\right)\right)-g(\psi(0,0))\right) d t d s\right| \\
& \quad+\left|\int_{-\frac{\varepsilon}{2}}^{\frac{\varepsilon}{2}} \int_{-\frac{\varepsilon}{2}}^{\frac{\varepsilon}{2}} \frac{\partial \gamma}{\partial r}(0, s, t) g(\psi(0,0))\left(\left\|\psi_{s} \times \psi_{t}\left(\frac{2 \delta s}{\varepsilon}, \frac{2 \delta t}{\varepsilon}\right)\right\|-\left\|\psi_{s} \times \psi_{t}(0,0)\right\|\right) d t d s\right| \\
& :=I+I I
\end{aligned}
$$

Using Cauchy- Schwartz inequality, a change of variables and the fact that $x_{0}=$ $\psi(0,0)$ is a Lebesgue point, we have

$$
\begin{aligned}
I & \leq\left(\int_{-\frac{\varepsilon}{2}}^{\frac{\varepsilon}{2}} \int_{-\frac{\varepsilon}{2}}^{\frac{\varepsilon}{2}}\left(\frac{\partial \gamma}{\partial r}(0, s, t)\right)^{2}\left\|\psi_{s} \times \psi_{t}\left(\frac{2 \delta s}{\varepsilon}, \frac{2 \delta t}{\varepsilon}\right)\right\| d t d s\right)^{\frac{1}{2}} \\
& \times\left(\int_{-\frac{\varepsilon}{2}}^{\frac{\varepsilon}{2}} \int_{-\frac{\varepsilon}{2}}^{\frac{\varepsilon}{2}}\left(g\left(\psi\left(\frac{2 \delta s}{\varepsilon}, \frac{2 \delta t}{\varepsilon}\right)\right)-g(\psi(0,0))\right)^{2}\left\|\psi_{s} \times \psi_{t}\left(\frac{2 \delta s}{\varepsilon}, \frac{2 \delta t}{\varepsilon}\right)\right\| d t d s\right)^{\frac{1}{2}} \\
& =\left(\int_{-\frac{\varepsilon}{2}}^{\frac{\varepsilon}{2}} \int_{-\frac{\varepsilon}{2}}^{\frac{\varepsilon}{2}}\left(\frac{\partial \gamma}{\partial r}(0, s, t)\right)^{2}\left\|\psi_{s} \times \psi_{t}\left(\frac{2 \delta s}{\varepsilon}, \frac{2 \delta t}{\varepsilon}\right)\right\| d t d s\right)^{\frac{1}{2}} \\
& \times\left(\frac{\varepsilon^{2}}{4 \delta^{2}} \int_{-\delta}^{\delta} \int_{-\delta}^{\delta}(g(\psi(\sigma, \tau))-g(\psi(0,0)))^{2}\left\|\psi_{s} \times \psi_{t}(\sigma, \tau)\right\| d t d s\right)^{\frac{1}{2}} \rightarrow 0 \text { as } \delta \rightarrow 0 .
\end{aligned}
$$

Next, as $\psi_{t}, \psi_{s}$ and $\frac{\partial \gamma}{\partial r}$ are continuous functions, we can use dominated convergence theorem to yield

$$
\begin{aligned}
I I & =\left|\int_{-\frac{\varepsilon}{2}}^{\frac{\varepsilon}{2}} \int_{-\frac{\varepsilon}{2}}^{\frac{\varepsilon}{2}} \frac{\partial \gamma}{\partial r}(0, s, t) g(\psi(0,0))\left(\left\|\psi_{s} \times \psi_{t}\left(\frac{2 \delta s}{\varepsilon}, \frac{2 \delta t}{\varepsilon}\right)\right\|-\left\|\psi_{s} \times \psi_{t}(0,0)\right\|\right) d t d s\right| \\
& \rightarrow 0 \text { as } \delta \rightarrow 0 .
\end{aligned}
$$

We therefore have

$$
g\left(x_{0}\right)\left\|\psi_{s} \times \psi_{t}(0,0)\right\| \int_{-\frac{\varepsilon}{2}}^{\frac{\varepsilon}{2}} \int_{-\frac{\varepsilon}{2}}^{\frac{\varepsilon}{2}} \frac{\partial \gamma}{\partial r}(0, s, t) d s d t=\lim _{\delta \rightarrow 0} \int_{\partial \Omega} \frac{\partial \varphi_{\delta}}{\partial \nu} g(x) d x=0
$$

as $\delta \rightarrow 0$.

Choosing $\gamma(r, s, t)=r \cdot a(s) b(t)$, where $a, b:(-\varepsilon, \varepsilon) \rightarrow \mathbb{R}$ are smooth and satisfy 
- $a(s), b(t) \geq 0$,

- $a(s), b(t)>0$ for $s, t \in\left(-\frac{\varepsilon}{3}, \frac{\varepsilon}{3}\right)$,

- $a(s), b(t)=0$ for $s, t \notin\left(-\frac{\varepsilon}{2}, \frac{\varepsilon}{2}\right)$.

Then we have

$$
\int_{-\frac{\varepsilon}{2}}^{\frac{\varepsilon}{2}} \int_{-\frac{\varepsilon}{2}}^{\frac{\varepsilon}{2}} \frac{\partial \gamma}{\partial r}(0, s, t) d s d t=\int_{-\frac{\varepsilon}{2}}^{\frac{\varepsilon}{2}} \int_{-\frac{\varepsilon}{2}}^{\frac{\varepsilon}{2}} a(s) b(t) d s d t>0
$$

and $g\left(x_{0}\right)=0$ as required.

We can now proceed with the proof of Theorem 1.1.

Proof. [of Theorem 1.1] First we consider $d=2$. Let $\bar{n}$ be a critical point of $\mathbb{E}$ in $\mathscr{A}$. By lemma $2.1 \bar{n}$ satisfies the equation

$$
\int_{\partial \Omega} \sum_{\beta, \alpha=1}^{d} \varphi_{\beta, \alpha} \bar{n}_{\alpha}\left(\nu_{\beta}-\bar{n}_{\beta}\langle\bar{n}, \nu\rangle\right) d S=0,
$$

for all $\varphi \in C^{\infty}\left(\Omega, \mathbb{R}^{3}\right)$ such that $\varphi=0$ on $\partial \Omega$. Let $\nu(x)=\left(\nu_{1}(x), \nu_{2}(x)\right)$ be the unit vector to $\partial \Omega$ at $x$ and $\tau(x)$ be a unit tangent to $\partial \Omega$ at $x$. Then at $x \in \partial \Omega$ we have

$$
\bar{n}=\langle\bar{n}, \nu\rangle \nu+\langle\bar{n}, \tau\rangle \tau
$$

plugging this into (2.3) gives

$$
0=\int_{\partial \Omega} \sum_{\beta, \alpha=1}^{d} \varphi_{\beta, \alpha}\left(\langle\bar{n}, \nu\rangle \nu_{\alpha}+\langle\bar{n}, \tau\rangle \tau_{\alpha}\right)\left(\nu_{\beta}-\bar{n}_{\beta}\langle\bar{n}, \nu\rangle\right) d S
$$

Since $\varphi=0$ on $\partial \Omega$ we have $\frac{\partial \varphi_{\beta}}{\partial \tau}=\varphi_{\beta, \alpha} \cdot \tau_{\alpha}=0$. Hence (2.3) simplifies to

$$
0=\int_{\partial \Omega} \sum_{\beta=1}^{d} \frac{\partial \varphi_{\beta}}{\partial \nu}\langle\bar{n}, \nu\rangle\left(\nu_{\beta}-\bar{n}_{\beta}\langle\bar{n}, \nu\rangle\right) d S .
$$

If $d=3$ then for any point $x_{0} \in \partial \Omega$ one can choose in a neighbourhood of $x_{0}$ two unit vector fields $P, Q$ such that $P \times Q=\nu$ and $\langle P, Q\rangle=0$. Then by writing

$$
\bar{n}=\langle\bar{n}, \nu\rangle \nu+\langle\bar{n}, P\rangle P+\langle\bar{n}, Q\rangle Q
$$


and using a partition of unity to use this into (2.3) we get that (2.5) holds for $d=3$. By setting $\varphi_{i} \equiv 0$ for $i \neq \beta$ in $(2.5)$, we get

$$
\int_{\partial \Omega} \frac{\partial \varphi}{\partial \nu}\langle\bar{n}, \nu\rangle\left(\nu_{\beta}-\bar{n}_{\beta}\langle\bar{n}, \nu\rangle\right) d S=0
$$

for $\beta=1,2,3$ and for all $\varphi \in C^{\infty}(\Omega, \mathbb{R})$ such that $\left.\varphi\right|_{\partial \Omega}=0$. By Lemma 2.2, we conclude that

$$
\langle\bar{n}, \nu\rangle\left(\nu_{\beta}-\bar{n}_{\beta}\langle\bar{n}, \nu\rangle\right)=0 \text { for } \beta=1, \ldots, d .
$$

Fix an arbitrary point $x \in \partial \Omega$. If $\langle\bar{n}(x), \nu(x)\rangle=0$ then we are done. Otherwise suppose $\langle\bar{n}(x), \nu(x)\rangle \neq 0$, and then we must have

$$
\left(\nu_{\beta}-\bar{n}_{\beta}\langle\bar{n}, \nu\rangle\right)=0 \text { for } \beta=1, \ldots, d .
$$

Since $\langle\bar{n}, \nu\rangle \neq 0$

$$
\bar{n}_{\beta}=\frac{\nu_{\beta}}{\langle\bar{n}, \nu\rangle} \text { for } \beta=1 \ldots d
$$

which implies

$$
\begin{aligned}
\langle\bar{n}, \nu\rangle & =\sum_{\beta=1}^{d} \frac{\nu_{\beta}}{\langle\bar{n}, \nu\rangle} \nu_{\beta} \\
& =\frac{\langle\nu, \nu\rangle}{\langle\bar{n}, \nu\rangle} \\
& =\frac{1}{\langle\bar{n}, \nu\rangle} .
\end{aligned}
$$

Hence

$$
\langle\bar{n}, \nu\rangle^{2}=1
$$

and therefore

$$
\langle\bar{n}, \nu\rangle= \pm 1
$$

\section{Partial regularity for tangential boundary con- ditions}

We restrict from now on our attention to the case when the boundary conditions are tangential, i.e. $n(x) \cdot \nu(x)=0$ for all $x \in \partial \Omega$, where $\nu$ is the outward pointing unit normal. 
We note that for any vector $v$ that is tangent to $\partial \Omega$ at $x$ we have

$$
\frac{d\langle n(x), \nu(x)\rangle}{d v}:=\sum_{\alpha=1}^{3} \frac{\partial\langle n(x), \nu(x)\rangle}{\partial x^{\alpha}} v^{\alpha}=0 .
$$

as $\langle n(x), \nu(x)\rangle$ is constant in the $v$ direction. That is tangential derivatives of $\langle n(x), \nu(x)\rangle$ are zero for all $x \in \partial \Omega$. Hence, as $n(x)$ is a tangent vector to $\partial \Omega$ at $x \in \partial \Omega$, we have

$$
\begin{aligned}
\sum_{\alpha, \beta=1}^{d} n^{\alpha} \frac{\partial n^{\beta}}{\partial x^{\alpha}} \nu^{\beta} & =\sum_{\alpha=1}^{d} n^{\alpha} \frac{\partial(n \cdot \nu)}{\partial x^{\alpha}}-\sum_{\alpha, \beta=1}^{d} \frac{\partial \nu^{\beta}}{\partial x^{\alpha}} n^{\beta} n^{\alpha} \\
& =-\sum_{\alpha, \beta=1}^{d} \frac{\partial \nu^{\beta}}{\partial x^{\alpha}} n^{\beta} n^{\alpha}
\end{aligned}
$$

Therefore, for maps with tangential boundary conditions we can write $\mathbb{E}$ as

$$
\mathbb{G}[n]:=\int_{\Omega} K \sum_{\alpha, \beta=1}^{d} \frac{\partial n^{\alpha}}{\partial x^{\beta}} \frac{\partial n^{\alpha}}{\partial x^{\beta}}-K_{13} \int_{\partial \Omega} \sum_{\alpha, \beta=1}^{d} \frac{\partial \nu^{\beta}}{\partial x^{\alpha}} n^{\beta} n^{\alpha} .
$$

This energy makes sense for maps in $W^{1,2}\left(\Omega, \mathbb{S}^{2}\right)$, and so we now focus on the slightly simpler task of minimizing $\mathbb{G}$ over the function space

$$
\mathcal{U}:=\left\{u \in W^{1,2}\left(\Omega, \mathbb{S}^{2}\right): \operatorname{Trace}(u) \in \mathcal{T}\right\}
$$

where

$$
\mathcal{T}:=\left\{\gamma \in H^{\frac{1}{2}}\left(\partial \Omega, \mathbb{S}^{2}\right): \gamma(x) \cdot \nu(x)=0 \text { for almost every } x \in \partial \Omega\right\} .
$$

Given the topological constraints associated with having tangential boundary conditions the first issue is to show that the function space $\mathcal{U}$ is non-empty. This will be addressed in the next subsection, while in the last subsection we will prove a partial regularity result for the minimizers.

\subsection{Function Space is non empty}

In this section we consider $\Omega$ to be a bounded domain of class $C^{2}$ and study whether or not the function space $\mathcal{U}$ defined through (3.1),(3.2) is non-empty.

If, for instance, $\partial \Omega$ is the torus then there exist smooth maps in $\mathcal{T}$ that have smooth extensions to the solid torus $\mathbb{B} \times \mathbb{S}^{1}$ and hence $\mathcal{U}$ would be non empty. However, if $\partial \Omega$ is $\mathbb{S}^{2}$ then the "Hairy Ball Theorem" tells us that there are no continuous maps 
in $\mathcal{T}$ and so it is not immediate that $\mathcal{U}$ is non empty. Fortunately, since $H^{\frac{1}{2}}$ in dimension two is larger than the space of continuous functions we are able to show that $\mathcal{T}$ and $\mathcal{U}$ are still non-empty even when $\partial \Omega$ a general $C^{2}$ surface. ${ }^{1}$

To this end we use an extension Theorem from [6] (stated as Theorem 3.6 in the following) which tells us that a function in $\mathcal{T}$ can be extended to a function in $\mathcal{U}$. This means to show $\mathcal{U}$ is non empty we only need to show that $\mathcal{T}$ is non empty. To do this we construct a function that belongs to $\mathcal{T}$ through a sequence of lemmas. We remark that a map $\gamma \in H^{\frac{1}{2}}\left(\partial \Omega, \mathbb{S}^{2}\right)$ is in $\mathcal{T}$ if and only if $\gamma(x) \in T_{x} \partial \Omega$ for almost every $x \in \partial \Omega$, where $T_{x} \partial \Omega$ is the tangent space to $\partial \Omega$ at $x$.

In Lemma 3.1 below we will give necessary and sufficient conditions to extend a vector field from the boundary of a manifold $N$ to its interior. Before we can state Lemma 3.1 we must first introduce some notation:

If $U$ is a $C^{2}$ manifold embedded in $\mathbb{R}^{d}$, let $T_{x} U$ be the tangent space to $U$ at $x \in U$. Let $g$ be a smooth vector field on $U$, i.e a smooth map $g: U \rightarrow \mathbb{R}^{d}$ such that $g(x) \in T_{x} U$ for every $x \in U$. Then let $i n d(g, U)$ denote the index of $g$ on $U$ (we refer the reader to [9],[18] or [3] for detailed properties of $i n d(g, U)$ ).

If $U$ is a manifold with boundary we define

$$
\partial_{-} U[g]:=\{x \in \partial U: g(x) \cdot \nu(x)<0\},
$$

where $\nu(x)$ is the outward-pointed unit normal to $U$ at $x$.

Furthermore, we recall that if $U$ is a compact surface then its Euler characteristic $\chi(U)$ can be related to its topological genus $k$ through the formula

$$
\chi(U)=2(1-k) .
$$

LEMma 3.1. Let $N$ be a $C^{1}$ manifold with boundary embedded in $\mathbb{R}^{d}$ and $g \in C^{\infty}\left(\partial N, \mathbb{R}^{d}\right)$ such that

$$
g(x) \in T_{x}(\partial N) \text { and }|g(x)|=1 .
$$

Then $g$ admits an extension to a continuous field $V: N \rightarrow \mathbb{R}^{d}$ such that, for every $x \in N, V(x) \in T_{x} N,|V(x)|=1$ and $\left.V\right|_{\partial N}=g$ if and only if

$$
\operatorname{ind}\left(g, \partial_{-} N[g]\right)=\chi(N) .
$$

Proof. Let $g \in C^{\infty}\left(\partial N, \mathbb{R}^{d}\right)$ such that (3.3) and (3.4) hold. Let $X$ be the topological double of $N$, that is, the manifold obtained by glueing two copies of $N$ along their boundaries (see [10] example 3.80 for a detailed construction of $X$ ). By modifying the value of $d$ if needed we can assume that $X$ is embedded in $\mathbb{R}^{d}$.

\footnotetext{
${ }^{1}$ We just need $C^{2}$ regularity for using Theorem 3.6, for all the other results of the section it would suffice to have $C^{1}$.
} 
Let $U \subset X$ be a tubular neighbourhood of $\partial N$ such that the nearest point projection $\pi: U \rightarrow \partial N$ is well defined. Let $\varphi: X \rightarrow \mathbb{R}$ be a smooth function such that $\left.\varphi\right|_{\partial N}=1$ and $\left.\varphi\right|_{X \backslash U}=0$. Then let $\tilde{G}: X \rightarrow \mathbb{R}^{d}$ be the extension of $g$ defined by

$$
\tilde{G}(x):=\left\{\begin{array}{l}
\operatorname{Proj}_{T_{x} X}(g(\pi(x))) \varphi(x) \text { for } x \in X \cap U, \\
0 \text { for } x \in X \backslash U,
\end{array}\right.
$$

where $\operatorname{Proj}_{T_{x} X}(y)$ is the projection of $y$ onto $T_{x} X$. As $0 \notin \tilde{G}(\partial N)$, by the Transitivity Theorem (see [2] Theorem 14.6), there exists a smooth tangent vector field $F$ on $X$ such that $F$ has finitely many zeros in $N$ and $\left.F\right|_{\partial N}=g$. Define $P_{\partial N} F$ to be the map

$$
P_{\partial N} F(x):=\operatorname{Proj}_{T_{x} \partial N}(F(x)) \text { for } x \in \partial N
$$

and for a continuous vector field $v: N \rightarrow \mathbb{R}^{d}$, define $\partial_{-} N[v]$ is to be the set

$$
\partial_{-} N[v]:=\{x \in \partial N: v(x) \cdot \nu(x)<0\} .
$$

By Morse's Index Formula (see [12]) and the stability of the index we have

$$
\begin{aligned}
\operatorname{ind}(F, N) & =\operatorname{ind}(\tilde{G}, N) \\
& =\chi(N)-i n d\left(P_{\partial N} \tilde{G}, \partial_{-} N\left[P_{\partial N} \tilde{G}\right]\right) \\
& =\chi(N)-i n d\left(g, \partial_{-} N[g]\right) \\
& =0 .
\end{aligned}
$$

We now just need to modify $F$ such that $|F|>0$ on $N$. Up to a continuous transformation, we can assume that all the zeros are contained in one coordinate patch $D \subset N$, with chart $\phi: \bar{D} \rightarrow \overline{B^{d}(0,1)}$ such that $\phi(\partial D)=\partial B^{d}(0,1)$, where $B^{d}(0,1)$ is the ball in $\mathbb{R}^{d}$ centred at 0 with radius 1 . Let $\tilde{F}: B^{d}(0,1) \rightarrow \mathbb{R}^{d}$ be the map defined as

$$
\tilde{F}(x):=F\left(\phi^{-1}(x)\right)
$$

and assume that $|\tilde{F}|>0$ in $B^{d}(0,1) \backslash B^{d}\left(0, \frac{1}{2}\right)$. Then,

$$
0=\operatorname{ind}\left(\tilde{F}, B^{d}(0,1)\right)=\operatorname{deg}\left(\frac{F}{|F|}, \partial D ; \mathbb{S}^{d-1}\right) .
$$

It can now be shown, as proved in [8], that there exists a harmonic field $\psi: B^{d}(0,1) \rightarrow$ $\mathbb{S}^{d-1}$ such that $\left.\psi\right|_{\partial B^{d}(0,1)}=\frac{\tilde{F}}{|\tilde{F}|}$. Finally, we define our extension:

$$
V(x)=\left\{\begin{array}{l}
\frac{F(x)}{|F(x)|} \text { if } x \in N \backslash D \\
\psi(\phi(x)) \text { if } x \in D .
\end{array}\right.
$$

$V$ is continuous and smooth everywhere apart from $\partial D$. 
Remark: Note that in the above construction we get a vector field on $N$ that is smooth almost everywhere.

We now relate the $H^{\frac{1}{2}}$ to a space whose norm is easier to compute, the $W^{1, p}$ space: LEMmA 3.2. Let $U \subset \mathbb{R}^{2}$ be an open set with $C^{1}$ boundary and $u \in W^{1, p}\left(U, \mathbb{S}^{2}\right)$ for $1<p<2$. Then $u \in H^{\frac{1}{2}}\left(U, \mathbb{S}^{2}\right)$.

In order to prove this we use the following Propositions from [20] (or [13]):

TheOREM 3.3. Let $p \in[1, \infty)$ and $s \in(0,1)$. Let $\Omega$ be an open set in $\mathbb{R}^{n}$ of class $C^{1}$ with bounded boundary and $u \in W^{1, p}(\Omega, \mathbb{R})$. Then

$$
\|u\|_{W^{s, p}(\Omega)} \leq C\|u\|_{W^{1, p}(\Omega)}
$$

for some positive constant $C=C(n, s, p) \geq 1$.

and

TheOREM 3.4. Let $p \in[1,+\infty), s \in(0,1)$ and $\Omega \subseteq \mathbb{R}^{n}$ be an open set of class $C^{1}$ with bounded boundary. Then $W^{s, p}(\Omega)$ is continuously embedded in $W^{s, p}\left(\mathbb{R}^{n}\right)$, namely there exists $C=C(n, \Omega)$ such that for any $u \in W^{s, p}(\Omega)$ there exists $\tilde{u} \in W^{s, p}\left(\mathbb{R}^{n}\right)$ such that $\left.\tilde{u}\right|_{\Omega}=u$ and

$$
\|\tilde{u}\|_{W^{s, p}\left(\mathbb{R}^{n}\right)} \leq C\|u\|_{W^{s, p}(\Omega)} .
$$

We also use the interpolation lemma from [11]

ThEOREM 3.5. For all $u \in W^{s, q}\left(\mathbb{R}^{n}\right) \cap L^{\infty}\left(\mathbb{R}^{n}\right)$ there holds the inequality

$$
\|u\|_{W^{\theta s, q / \theta}\left(\mathbb{R}^{n}\right)} \leq c(n)\left(\frac{q}{q-1}\right)^{\theta}\left(\frac{1-s}{1-\theta}\right)^{\frac{\theta}{q}}\|u\|_{W^{s, q}\left(\mathbb{R}^{n}\right)}^{\theta}\|u\|_{L^{\infty}}^{1-\theta}
$$

where $0<s<1,1<q<\infty$, and $0<\theta<1$.

We can now prove Lemma 3.2.

Proof. [Lemma 3.2] Let $1<p<2$ and $u \in W^{1, p}(U)$. By Theorem 3.3 we have that $u \in W^{\frac{2}{3}, p}(U)$. Let $\tilde{u} \in W^{\frac{2}{3}, p}\left(\mathbb{R}^{2}\right)$ be the extension given by Theorem 3.4. By setting $s=\frac{2}{3}, \theta=\frac{3}{4}$ and $q=\frac{3}{2}$ in Theorem 3.5 we have

$$
\begin{aligned}
& \|u\|_{H^{\frac{1}{2}(U)}} \leq\|\tilde{u}\|_{H^{\frac{1}{2}\left(\mathbb{R}^{2}\right)}}=\|\tilde{u}\|_{W^{\frac{2}{3} \cdot \frac{3}{4}, 2\left(\mathbb{R}^{2}\right)}} \leq C\|\tilde{u}\|_{W^{\frac{2}{3}, \frac{3}{2}\left(\mathbb{R}^{2}\right)}}^{\frac{3}{4}}\|u\|_{L^{\infty}\left(\mathbb{R}^{2}\right)}^{\frac{1}{4}} \\
& \leq C\|u\|_{W^{\frac{2}{3}, \frac{3}{2}(U)}}^{\frac{3}{4}}\|u\|_{L^{\infty}\left(\mathbb{R}^{2}\right)}^{\frac{1}{4}} \leq C\|u\|_{W^{1, \frac{3}{2}(U)}}^{\frac{3}{4}}\|u\|_{L^{\infty}\left(\mathbb{R}^{2}\right)}^{\frac{1}{4}}<\infty .
\end{aligned}
$$


In order to prove Proposition 1.2 we use an extension theorem from [6], which we state here for the reader's convenience.

TheOrem 3.6. If $1<p<m, \Omega$ is a bounded $C^{2}$ domain in $\mathbb{R}^{m}$, and $N$ is a compact $C^{2}$ submanifold of $\mathbb{R}^{k}$ with $\pi_{0}(N)=\pi_{1}(N)=\ldots \pi_{[p]-1}(N)=0$, then any function $\eta \in W^{1-1 / p, p}(\partial \Omega, N)$ admits an extension $\omega \in W^{1, p}(\Omega, N)$.

We are now in a position to prove Proposition 1.2

Proof. [Proposition 1.2] Let $\Omega$ be a $C^{2}$ domain such that $\partial \Omega$ is a surface of genus $k$. Then we have that

$$
\chi(\partial \Omega)=2(1-k) .
$$

We first show that the map $\varphi: B^{2}(0,1) \rightarrow \mathbb{S}^{1}$ defined by

$$
\varphi\left(x^{1}, x^{2}\right)=\left(\frac{-x^{2}}{\sqrt{\left(x^{1}\right)^{2}+\left(x^{2}\right)^{2}}}, \frac{x^{1}}{\sqrt{\left(x^{1}\right)^{2}+\left(x^{2}\right)^{2}}}\right)
$$

is in $W^{1, p}\left(B^{2}(0,1), \mathbb{S}^{1}\right)$. Then $|\nabla \varphi|=\frac{1}{|x|}$ and we have: thus

$$
\int_{B^{2}(0,1)}|\nabla \varphi|^{p} d x \leq C \int_{0}^{1} r^{1-p} d r
$$

which is finite for $1<p<2$, hence $\varphi \in W^{1, p}\left(B^{2}(0,1)\right.$, $\left.\mathbb{S}^{1}\right)$ for $1<p<2$.

For $i=1 \ldots|\chi(\partial \Omega)|$ let $\left(U_{i}, \psi_{i}\right)$ be coordinate patches on $\partial \Omega$ such that $\bigcap_{i=1}^{|\chi(\partial \Omega)|} U_{i}=$ $\emptyset$. Using

$$
\varphi\left(x^{1}, x^{2}\right)=\left(\frac{-x^{2}}{\sqrt{\left(x^{1}\right)^{2}+\left(x^{2}\right)^{2}}}, \frac{x^{1}}{\sqrt{\left(x^{1}\right)^{2}+\left(x^{2}\right)^{2}}}\right),
$$

or

$$
\tilde{\varphi}\left(x^{1}, x^{2}\right)=\left(\frac{x^{2}}{\sqrt{\left(x^{1}\right)^{2}+\left(x^{2}\right)^{2}}}, \frac{-x^{1}}{\sqrt{\left(x^{1}\right)^{2}+\left(x^{2}\right)^{2}}}\right)
$$

we can put a unit vector field, $v_{i}$, on each $U_{i}$ such that the map $V: \bigcup U_{i} \rightarrow \mathbb{S}^{2}$ defined by

$$
v(x):=v_{i}(x) \text { if } x \in U_{i}
$$

satisfies

$$
\left.\operatorname{ind}\left(\left.v\right|_{\partial\left(\cup U_{i}\right)}, \partial_{-}\left(\partial \Omega \backslash \cup U_{i}\right)\right)[v]\right)=\chi(\partial \Omega) .
$$

Using Lemma 3.1 we can now extend $V$ to a vector field on $\partial \Omega$ that is smooth on $\partial \Omega \backslash \bigcup U_{i}$. Since $\varphi$ and $\tilde{\varphi}$ are both in $W^{1, p}\left(\partial \Omega, \mathbb{S}^{2}\right)$ for $1<p<2$ the map $V$ is in $W^{1, p}\left(\partial \Omega, \mathbb{S}^{2}\right)$. Then by Lemma 3.2 we have that $V \in H^{\frac{1}{2}}\left(\partial \Omega, \mathbb{S}^{2}\right)$, it follows that $V \in \mathcal{T}$ and hence $\mathcal{T}$ is non empty. Using Theorem 3.6 we can now conclude that the function space $\mathcal{T}$ is non empty. 


\subsection{Regularity of Minimizers}

We prove that a minimizer, $u$, of $\mathbb{G}$ in $\mathcal{U}$ is continuous on $\Omega \backslash Z$, where $Z$ is some subset of $\Omega$ with zero one-dimensional Hausdorff measure. We show that for $x \in \Omega \backslash Z$ the rescaled energy

$$
\mathbb{E}_{r}[u]:=r^{-1} \int_{\Omega \cap C(a, r)}|\nabla u|^{2} d x
$$

decays suitably fast as $r$ tends to 0 . Continuity of $u$ then follows by Morrey's Lemma (see for example [19] Chapter 18). Our proof of the energy decay is based on the work of Schoen and Uhlenbeck [16] and the papers by Hardt and Lin [6], [7].

We give a brief outline of the proof here. For a given domain $\Omega$ we construct a map $Q: \Omega \rightarrow S O(3)$ (depending only on $\partial \Omega$ ) such that for an arbitrary map $u \in \mathcal{U}$ we have that $Q(x) u(x)$ lies on the equator of $\mathbb{S}^{2}$ for almost all $x \in \partial \Omega$. This transforms our tangential boundary conditions into a partially constrained boundary condition as in [7], so we can adapt some of the results there in order to prove a Hybrid inequality (Lemma 3.11). The proof of the energy decay (Lemma 3.12 and Theorem 3.13) is then done by contradiction. We assume there exists a sequence of minimizers, $u_{i}$, and domains, $\Omega_{i}$, that do not have energy decay but do satisfy $\varepsilon_{i}=\int_{\Omega_{i}}\left|\nabla u_{i}\right|^{2} d x \rightarrow 0$ as $i \rightarrow \infty$. We then form the blow-up sequence $\varepsilon_{i}^{-1}\left(u_{i}-\overline{\bar{u}}_{i}\right)$ that converges weakly to a blow up function $v$. We then prove some estimates on $v$ by showing it is harmonic. These estimates are then transferred to the $u_{i}$ using the Hybrid inequality in order to get a contradiction.

\subsubsection{Scaling and notations}

In order to apply Morrey's Lemma we must investigate how our energy scales. For $x \in \mathbb{R}^{3}$ define the cylinder $C(x, r):=\left\{y \in \mathbb{R}^{3}:\left|\left(y^{1}, y^{2}\right)-\left(x^{1}, x^{2}\right)\right|<r,\left|y^{3}-x^{3}\right|<r\right\}$. Let

$$
\varphi \in C^{2}\left(\mathbb{R}^{2}, \mathbb{R}\right) \text { with } \varphi(0)=0=|\nabla \varphi(0)|, \operatorname{Lip}(\varphi) \leq 1
$$

and define

$$
\begin{aligned}
\Omega_{\varphi}: & =\left\{\left(x^{1}, x^{2}, x^{3}\right) \in C(0,1): x^{3}<\varphi\left(x^{1}, x^{2}\right)\right\} \\
& =\left\{x \in \mathbb{R}^{3}:\left|\left(x^{1}, x^{2}\right)\right|<1 \text { and }-1<x^{3}<\varphi\left(x^{1}, x^{2}\right)\right\} .
\end{aligned}
$$

For a domain $\Omega \subset \mathbb{R}^{3}, a \in \partial \Omega$ there exists $R>0, h \in S O(3)$ and $\varphi_{R, a} \in C^{2}\left(\mathbb{R}^{2}, \mathbb{R}\right)$ such that $\varphi_{R, a}(0)=0=\left|\nabla \varphi_{R, a}(0)\right|, \operatorname{Lip}\left(\varphi_{R, a}\right) \leq 1$ and

$$
\Omega_{\varphi_{R, a}}=\left\{h^{-1}[(y-a) / R]: y \in C(a, R) \cap \Omega\right\} .
$$


For $0<r \leq R$, let $\varphi_{r, a}=\varphi_{R, a}\left(\frac{r x}{R}\right)$, then for $u \in \mathcal{U}$, the expression $n_{r, a}(x)=$ $n[r h(x)+a]$ defines a function in $W^{1,2}\left(\Omega_{\varphi_{r, a}}, \mathbb{S}^{2}\right)$ whose trace, $\gamma$, on $\partial \Omega_{\varphi_{r, a}} \backslash \partial C(0,1)$ satisfies $\gamma(x) \cdot(h \cdot \nu(x))+a)=0$ almost everywhere. We note that

$$
\begin{gathered}
\mathbb{E}_{1}\left(u_{r, a}\right)=\int_{\Omega_{\varphi} \cap C(0,1)}\left|\nabla u_{r, a}(x)\right|^{2} d x=r^{-1} \int_{\Omega_{\varphi} \cap C(a, r)}|\nabla u|^{2} d x \\
\operatorname{Lip}\left(\varphi_{r, a}\right)=\operatorname{Lip}\left(\varphi_{R, a}\right) R^{-1} r \\
\left\|\varphi_{r, a}\right\|_{C^{2}}:=\max _{|\alpha|=2} \sup _{x \in \mathbb{R}^{2}}\left|\frac{\partial^{|\alpha|} \varphi_{r, a}(x)}{\partial x^{\alpha}}\right|=R^{-2} r^{2}\left\|\varphi_{R, a}\right\|_{C^{2}} .
\end{gathered}
$$

For convenience we collect the notations of various domains we will use

$$
\begin{array}{ll}
\Omega_{\varphi}:=\left\{\left(x^{1}, x^{2}, x^{3}\right) \in C(0,1): x^{3}<\varphi\left(x^{1}, x^{2}\right)\right\} & \Omega_{0}:=\left\{\left(x^{1}, x^{2}, x^{3}\right) \in C(0,1): x^{3}<0\right\} \\
G_{\varphi}:=\partial \Omega_{\varphi} \backslash \partial C(0,1) & G_{0}:=\partial \Omega_{0} \backslash \partial C(0,1) \\
H_{\varphi}:=\partial \Omega_{\varphi} \cap \partial C(0,1) & H_{0}:=\partial \Omega_{0} \cap \partial C(0,1) \\
\mathbb{B}_{r}:=\left\{x \in \mathbb{R}^{3} ;|x| \leq r\right\} & \mathbb{B}:=B^{3}(0,1)
\end{array}
$$

Using these notations we define the energy, $\mathbb{G}_{\varphi}$, for a map $u \in W^{1,2}\left(\Omega_{\varphi}, \mathbb{S}^{2}\right)$ to be

$$
\mathbb{G}_{\varphi}[u]:=\int_{\Omega_{\varphi}}|\nabla u|^{2} d x-K_{13} \int_{G_{\varphi}} u^{\alpha} u^{\beta} \frac{\partial \nu^{\beta}}{\partial x^{\alpha}} d x .
$$

\subsubsection{A Useful Projection}

Given $\varphi$ as above we will construct a map

$$
Q: \Omega_{\varphi} \rightarrow S O(3)
$$

such that if

$$
u \in \mathcal{U}_{\varphi}:=\left\{u \in W^{1,2}\left(\Omega_{\varphi}, \mathbb{S}^{2}\right): \operatorname{Trace}(u) \in \mathcal{T}_{\varphi}\right\}
$$

where

$$
\mathcal{T}_{\varphi}=\left\{\gamma \in H^{\frac{1}{2}}\left(G_{\varphi}, \mathbb{S}^{2}\right): \gamma(x) \cdot \nu(x)=0 \text { almost everywhere }\right\} .
$$

then the map $w(x):=Q(x) u(x)$ is in the function space

$$
\mathscr{E}_{\varphi}=\left\{v \in W^{1,2}\left(\Omega_{\varphi}, \mathbb{S}^{2}\right): \operatorname{Trace}(v) \in \tilde{\mathbb{S}}^{1}\right\}
$$

where $\tilde{\mathbb{S}}^{1}=\left\{\left(x^{1}, x^{2}, x^{3}\right) \in \mathbb{S}^{2}: x^{3}=0\right\}$.

We construct $Q$ as follows: let $x \in G_{\varphi}$ and let $\nu(x)$ be the unit norm to $G_{\varphi}$ at $x$. Define $Q(x)$ to be the rotation about the axis $\nu(x) \times(0,0,1)$ through the angle $\tau$ given by $\cos (\tau)=\nu(x) \cdot(0,0,1)$. Explicitly $Q$ is given by 


$$
Q(x):=\left[\begin{array}{ccc}
\frac{\varphi_{x^{2}}^{2}+\varphi_{x^{1}}^{2} \cos (\tau)}{\varphi_{x^{1}}^{2}+\varphi_{x^{2}}^{2}} & -\frac{\varphi_{x^{1}} \varphi_{x^{2}}(1-\cos (\tau))}{\varphi_{x^{1}}^{2}+\varphi_{x^{2}}^{2}} & \frac{\varphi_{x^{1}} \sin (\tau)}{\left(\varphi_{x^{1}}^{2}+\varphi_{x^{2}}^{2}\right)^{\frac{1}{2}}} \\
-\frac{\varphi_{x^{1}} \varphi_{x^{2}}(1-\cos (\tau))}{\varphi_{x^{1}}^{2}+\varphi_{x^{2}}^{2}} & \frac{\varphi_{x^{1}}^{2}+\varphi_{x^{2}}^{2} \cos (\tau)}{\varphi_{x^{1}}^{2}+\varphi_{x^{2}}^{2}} & \frac{\varphi_{x^{2}} \sin (\tau)}{\left(\varphi_{x^{1}}^{2}+\varphi_{x^{2}}^{2}\right)^{\frac{1}{2}}} \\
-\frac{\varphi_{x^{1}} \sin (\tau)}{\left(\varphi_{x^{1}}^{2}+\varphi_{x^{2}}^{2}\right)^{\frac{1}{2}}} & -\frac{\varphi_{x^{2}} \sin (\tau)}{\left(\varphi_{x^{1}}^{2}+\varphi_{x^{2}}^{2}\right)^{\frac{1}{2}}} & \cos (\tau)
\end{array}\right]
$$

where $\varphi_{x_{i}}=\frac{\partial \varphi}{\partial x_{i}}, \cos (\tau)=\left(\varphi_{x^{1}}^{2}+\varphi_{x^{2}}^{2}+1\right)^{-\frac{1}{2}}$ and $\sin (\tau)=\sqrt{\frac{\varphi_{x^{1}}^{2}+\varphi_{x^{2}}^{2}}{\varphi_{x^{1}}^{2}+\varphi_{x^{2}}^{2}+1}}$. Then for $x=\left(x^{1}, x^{2}, x^{3}\right) \in \Omega_{\varphi}$ define

$$
Q(x)=Q\left(\left(x^{1}, x^{2}, \varphi\left(x^{1}, x^{2}\right)\right)\right) .
$$

It is straightforward to check that $Q \in C^{1}\left(\Omega_{\varphi}, S O(3)\right)$. We now find some bounds on the entries of $Q$ that will be useful throughout our proof.

LEMmA 3.7. Let $Q, \varphi$ be defined as above, then we have

$$
\sup \left\{|Q(x) n-n|: x \in \Omega_{\varphi}, n \in \mathbb{S}^{2}\right\} \leq 9 \operatorname{Lip}(\varphi) .
$$

Proof. Let $x \in G_{\varphi}$ and $n \in \mathbb{S}^{2}$. We have $|Q(x) n-n| \leq \sum_{i=1}^{3}\left|(Q(x) n-n)_{i}\right|$, where $(Q(x) n-n)_{i}$ is the $i^{t h}$ component of $Q(x) n-n$. We find bounds on each component individually.

$\left|(Q(x) n-n)_{1}\right|=\left|\left(\frac{\varphi_{x^{2}}^{2}+\varphi_{x^{1}}^{2} \cos (\tau)-\varphi_{x^{1}}^{2}-\varphi_{x^{2}}^{2}}{\varphi_{x^{1}}^{2}+\varphi_{x^{2}}^{2}}\right) n_{1}-\frac{\varphi_{x^{1}} \varphi_{x^{2}}(1-\cos (\tau)) n_{2}}{\varphi_{x^{1}}^{2}+\varphi_{x^{2}}^{2}}+\varphi_{x_{1}} \cos (\tau) n_{3}\right|$

Writing $\varphi_{x^{1}}(x)=r \cos (t), \varphi_{x^{2}}(x)=r \sin (t)$ for appropriate $r \in(0, \infty), t \in[0,2 \pi)$ we have

$$
\begin{aligned}
\left|(Q(x) n-n)_{1}\right| & \leq\left|\frac{r^{2} \cos ^{2}(t)\left(\left(r^{2}+1\right)^{-\frac{1}{2}}-1\right)}{r^{2}}\right|+\left|\frac{r^{2} \cos (t) \sin (t)\left(1-\left(r^{2}+1\right)^{-\frac{1}{2}}\right)}{r^{2}}\right|+|r| \\
& \leq 2 r+r \\
& \leq 3 \operatorname{Lip}(\varphi)
\end{aligned}
$$

We can get a similar estimate for $(Q(x) n-n)_{2}$ and $(Q(x) n-n)_{3}$, hence

$$
|Q(x) n-n| \leq 9 \operatorname{Lip}(\varphi)
$$


LEMMA 3.8.

$$
\sup \left\{\left|\frac{\partial Q_{i j}(x)}{\partial x^{k}}\right|: i, j, k \in\{1,2,3\}, x \in \Omega_{\varphi}\right\}<6\|\varphi\|_{C^{2}}
$$

Proof. Let $x \in \Omega_{\varphi}$, we find bounds on each $\frac{\partial Q_{i j}(x)}{\partial x^{k}}$, write $\varphi_{x^{1}}(x)=r \sin (t), \varphi_{x^{2}}(x)=$ $r \cos (t)$.

$$
\begin{aligned}
&\left|\frac{\partial Q_{11}(x)}{\partial x^{k}}\right|=\left|\frac{\varphi_{x^{1}}^{2} \frac{\partial \cos (\tau)}{\partial x^{k}}}{\left(\varphi_{x^{1}}^{2}+\varphi_{x^{2}}^{2}\right)}+2\left[\frac{\varphi_{x^{2} x^{k}} \varphi_{x^{1}}^{2} \varphi_{x^{2}}(1-\cos (\tau))+\varphi_{x^{1} x^{\alpha}} \varphi_{x^{1}} \varphi_{x^{2}}^{2}(\cos (\tau)-1)}{\left(\varphi_{x^{1}}^{2}+\varphi_{x^{2}}^{2}\right)^{2}}\right]\right| \\
& \leq\left|\frac{\varphi_{x^{1}}^{2}\left(\varphi_{x^{1}} \varphi_{x^{1} x^{k}}+\varphi_{x^{2}} \varphi_{x^{2} x^{k}}\right)}{\left(\varphi_{x^{1}}^{2}+\varphi_{x^{2}}^{2}\right)\left(\varphi_{x^{1}}^{2}+\varphi_{x^{2}}^{2}+1\right)^{\frac{3}{2}}}\right|+2\|\varphi\|_{C^{2}}\left[\left|\frac{\sin ^{2}(t) \cos (t)(1-\cos (\tau))}{r}\right|\right. \\
&\left.\quad+\left|\frac{\sin (t) \cos (t)(1-\cos (\tau))}{r}\right|\right] \\
& \leq 2\|\varphi\|_{C^{2}} \operatorname{Lip}(\varphi)+4\|\varphi\|_{C^{2}} \operatorname{Lip}(\varphi)
\end{aligned}
$$

By the symmetry of $Q, \frac{\partial Q_{22}}{\partial x^{k}}$ will have the same bound.

Next, bounding $\frac{\partial Q_{12}}{\partial x^{k}}$;

$$
\begin{aligned}
\left|\frac{\partial Q_{12}}{\partial x^{k}}\right| & =\mid \frac{\left(\varphi_{x^{1} x^{k}} \varphi_{x^{2}}+\varphi_{x^{1}} \varphi_{x^{2} x^{k}}\right)(\cos (\tau)-1)}{\varphi_{x^{1}}^{2}+\varphi_{x^{2}}^{2}}+2 \frac{\varphi_{x^{1}} \varphi_{x^{2}}\left(\varphi_{x^{1}} \varphi_{x^{1} x^{k}}+\varphi_{x^{2}} \varphi_{x^{2} x^{k}}\right)(1-\cos (\tau))}{\left(\varphi_{x^{1}}^{2}+\varphi_{x^{2}}^{2}\right)^{2}} \\
& \quad-\frac{\varphi_{x^{1}} \varphi_{x^{2}}\left(\varphi_{x^{1}} \varphi_{x^{1} x^{k}}+\varphi_{x^{2}} \varphi_{x^{2} x^{k}}\right)}{\left(\varphi_{x^{1}}^{2}+\varphi_{x^{2}}^{2}\right)\left(\varphi_{x^{1}}^{2}+\varphi_{x^{2}}^{2}+1\right)^{\frac{3}{2}}} \mid \\
& \leq\|\varphi\|_{C^{2}} r^{2}\left(\left|\frac{r \cos (t)}{r^{2}}\right|+\left|\frac{r \sin (t)}{r^{2}}\right|\right)+2 r^{4}\|\varphi\|_{C^{2}}\left(\left|\frac{r \cos (t)}{r^{4}}\right|+\left|\frac{r \sin (t)}{r^{4}}\right|\right) \\
& \leq 6\|\varphi\|_{C^{2}} \operatorname{Lip}(\varphi) \\
& \leq 6\|\varphi\|_{C^{2}} .
\end{aligned}
$$

Again, by the symmetry of $Q, \frac{\partial Q_{21}}{\partial x^{k}}$ will have the same bounds. We have

$$
\begin{aligned}
\left|\frac{\partial Q_{13}}{\partial x^{k}}\right| & =\left|\frac{\varphi_{x^{1} x^{k}}}{\left(\varphi_{x^{1}}^{2}+\varphi_{x^{2}}^{2}+1\right)^{\frac{1}{2}}}-\frac{\varphi_{x^{1}}\left(\varphi_{x^{1}} \varphi_{x^{1} x^{k}}+\varphi_{x^{2}} \varphi_{x^{2} x^{k}}\right)}{\left(\varphi_{x^{1}}^{2}+\varphi_{x^{2}}^{2}+1\right)^{\frac{3}{2}}}\right| \\
& \leq\|\varphi\|_{C^{2}}+2\|\varphi\|_{C^{2}} \operatorname{Lip}(\varphi)^{2} \\
& \leq 3\|\varphi\|_{C^{2}}
\end{aligned}
$$


and $\frac{\partial Q_{23}}{\partial x^{k}}, \frac{\partial Q_{31}}{\partial x^{k}}, \frac{\partial Q_{32}}{\partial x^{k}}$ will have the same bounds. Finally,

$$
\begin{aligned}
\left|\frac{\partial Q_{33}}{\partial x^{k}}\right| & =\left|\frac{\varphi_{x^{1}} \varphi_{x^{1} x^{k}}+\varphi_{x^{2}} \varphi_{x^{2} x^{k}}}{\left(\varphi_{x^{1}}^{2}+\varphi_{x^{2}}^{2}+1\right)^{\frac{3}{2}}}\right| \\
& \leq 2 \operatorname{Lip}(\varphi)\|\varphi\|_{C^{2}} \\
& \leq 2\|\varphi\|_{C^{2}} .
\end{aligned}
$$

This gives the result with $K=6$.

LEMMA 3.9.

$$
\sup \left\{Q_{i j}(x): i, j \in\{1,2,3\} \text { and } x \in \Omega_{\varphi}\right\}=1
$$

Proof. First we note that $Q_{11}(0)=1$ and so $1 \leq \sup \left\{Q_{i j}(x): i, j \in\{1,2,3\}, x \in \Omega_{\varphi}\right\}$. Next we calculate

$$
\begin{gathered}
\left|Q_{11}(x)\right| \leq \frac{\varphi_{x^{2}}^{2}}{\varphi_{x^{1}}^{2}+\varphi_{x^{2}}^{2}}+\frac{\varphi_{x^{1}}^{2}}{\varphi_{x^{1}}^{2}+\varphi_{x^{2}}^{2}}=1 \\
\left|Q_{12}(x)\right| \leq\left|\frac{\varphi_{x^{2}} \varphi_{x^{1}}(1-\cos (\tau))}{\varphi_{x^{1}}^{2}+\varphi_{x^{2}}^{2}}\right| \leq\left|\frac{2 \varphi_{x^{2}} \varphi_{x^{1}}}{\varphi_{x^{1}}^{2}+\varphi_{x^{2}}^{2}}\right| \leq 1 \\
\left|Q_{13}(x)\right| \leq\left|\frac{\varphi_{x^{1}}}{\left(\varphi_{x^{1}}^{2}+\varphi_{x^{2}}^{2}+1\right)^{\frac{1}{2}}}\right| \leq\left|\varphi_{x^{1}}\right| \leq 1 \\
\left|Q_{33}(x)\right|=|\cos (\tau)| \leq 1
\end{gathered}
$$

The other terms are similar or the same.

\subsubsection{Proof of Partial Regularity}

We will use the projection $Q$ and the following extension Lemma from [7] in order to prove a Hybrid Inequality. Note that the following lemma is stated for balls rather than cylinders.

LEMMA 3.10. There are positive constants $\delta, q$ and $c$ such that, if $0<\varepsilon<1, \xi \in \mathbb{R}^{3}$, and $\eta \in W^{1,2}\left(\Omega_{\varphi} \cap \partial \mathbb{B}, \mathbb{S}^{2}\right)$ satisfies the small oscillation condition

$$
\left[\int_{\Omega_{\varphi} \cap \partial \mathbb{B}}\left|\nabla_{t a n} \eta\right|^{2} d \mathcal{H}^{2}\right]\left[\int_{\Omega_{\varphi} \cap \partial \mathbb{B}}|\eta-\xi|^{2} d \mathcal{H}^{2}+\int_{\partial\left(\Omega_{\varphi} \cap \partial \mathbb{B}\right)}|\eta-\xi|^{2} d \mathcal{H}^{1}\right] \leq \delta^{2} \varepsilon^{q}
$$

and if $\left.\eta\right|_{\partial \Omega_{\varphi} \cap \partial \mathbb{B}}$ has image in $\mathbb{S}^{1}$, then there exists a function $\omega \in W^{1,2}\left(\Omega_{\varphi} \cap \mathbb{B}, \mathbb{S}^{2}\right)$, $\left.\omega\right|_{\Omega_{\varphi} \cap \partial \mathbb{B}}=\eta,\left.\omega\right|_{G_{\varphi}}$ has image in $\mathbb{S}^{1}$ and

$$
\int_{\Omega_{\varphi} \cap \mathbb{B}}|\nabla \omega|^{2} d x \leq \varepsilon \int_{\Omega_{\varphi} \cap \partial \mathbb{B}}\left|\nabla_{t a n} \eta\right|^{2} d \mathcal{H}^{2}+c \varepsilon^{-q}\left[\int_{\Omega_{\varphi} \cap \partial \mathbb{B}}|\eta-\xi|^{2} d \mathcal{H}^{2}+\int_{\partial\left(\Omega_{\varphi} \cap \partial \mathbb{B}\right)}|\eta-\xi|^{2} d \mathcal{H}^{1}\right] .
$$


Proof. For a proof see Theorem 3.1 in [7].

We can now prove our main ingredient:

Lemma 3.11. [Hybrid inequality] There exists positive constants $c_{1}, c_{2}$ and $q$ such that if $0 \leq \lambda \leq 1, \varphi$ is as above, $u$ is a minimizer of $\mathbb{G}_{\varphi}$ amongst maps in $\mathcal{U}_{\varphi}$ with fixed trace on $H_{\varphi}$ such that $\int_{\Omega_{\varphi}}|\nabla u|^{2} d x<c_{1} \lambda^{\frac{q}{2}}, \operatorname{Lip}(\varphi) \leq c_{1} \lambda^{\frac{q}{2}}$ and $\|\varphi\|_{C^{2}} \leq c_{1} \lambda^{\frac{q}{2}}$, then

$$
\begin{aligned}
\left(\frac{1}{2}\right)^{-1} \int_{\Omega_{\varphi} \cap C\left(0, \frac{1}{4}\right)}|\nabla u|^{2} d x \leq & \lambda\left(\|\varphi\|_{C^{2}}^{2}+\int_{\Omega_{\varphi} \cap C(0,1)}|\nabla u|^{2} d x\right) \\
& +\lambda^{-q} C_{2}\left(\operatorname{Lip}(\varphi)^{2}+\int_{\Omega_{\varphi} \cap C(0,1)}|u-\overline{\bar{u}}|^{2} d x\right) \\
& +\lambda^{-q} C_{2} \int_{G_{\varphi} \cap C(0,1)}|u-\overline{\bar{u}}|^{2} d \mathcal{H}^{2}+c_{2}\|\varphi\|_{C^{2}}^{2} .
\end{aligned}
$$

where $\overline{\bar{u}}:=\mathcal{H}^{2}\left(G_{\varphi}\right)^{-1} \int_{G_{\varphi}} u d \mathcal{H}^{2}$.

Proof. As we have the set inclusions $C\left(0, \frac{1}{4}\right) \subset B^{3}\left(0, \frac{1}{2}\right)$ and $\mathbb{B} \subset C(0,1)$ it suffices to prove the inequality

$$
\begin{aligned}
\left(\frac{1}{2}\right)^{-1} \int_{\Omega_{\varphi} \cap \mathbb{B}_{\frac{1}{2}}}|\nabla u|^{2} d x \leq & \lambda\left(\|\varphi\|_{C^{2}}^{2}+\int_{\Omega_{\varphi} \cap \mathbb{B}}|\nabla u|^{2} d x\right) \\
& +\lambda^{-q} c_{2}\left(\operatorname{Lip}(\varphi)^{2}+\int_{\Omega_{\varphi} \cap \mathbb{B}}|u-\overline{\bar{u}}|^{2} d x\right) \\
& +\lambda^{-q} c_{2} \int_{G_{\varphi} \cap \mathbb{B}}|u-\overline{\bar{u}}|^{2} d \mathcal{H}^{2}+c_{2}\|\varphi\|_{C^{2}}^{2} .
\end{aligned}
$$

Let $u$ be a map satisfying the assumptions of the Lemma. We aim to apply Lemma 3.10 to the map $Q(x) u(x)$. In order to do this we must check the map 
$Q(x) u(x)$ satisfies the small oscillation condition (3.7). We first bound

$$
\begin{aligned}
|\nabla(Q(x) u(x))|^{2} & =\sum_{i, k, l=1}^{3}\left(\frac{\partial\left(Q_{i j} u^{j}\right)}{\partial x^{k}}\right)^{2} \\
& =\sum_{i, j, k}\left(\frac{\partial Q_{i j}}{\partial x^{k}} u^{j}+\frac{\partial u^{j}}{\partial x^{k}} Q_{i, j}\right)^{2} \\
& \leq 2 \sum_{i, j, k}\left[\left(\frac{\partial Q_{i j}}{\partial x^{k}} u^{j}\right)^{2}+\left(\frac{\partial u^{j}}{\partial x^{k}} Q_{i j}\right)^{2}\right] \\
& \leq 2 \sum_{i, k}\left[\sum_{j=1}^{3}\left(\frac{\partial Q_{i, j}}{\partial x^{k}}\right)^{2} \sum_{l=1}^{3}\left(u^{l}\right)^{2}+\sum_{m=1}^{3}\left(\frac{\partial u^{m}}{\partial x^{k}}\right)^{2} \sum_{n=1}^{3}\left(Q_{i n}\right)^{2}\right] \\
& \leq 18\left(\|\varphi\|_{C^{2}}^{2}+|\nabla u|^{2}\right)
\end{aligned}
$$

by Lemma 3.8 and 3.9.

As in [5], we note that for an increasing function $\eta:[0,1] \rightarrow \mathbb{R}$ we have

$$
\mathcal{L}^{1}\left(\left\{s: \eta^{\prime}(s) \geq 8(\eta(1)-\eta(0))\right\}\right) \leq \frac{1}{8} .
$$

Hence we can find $\sigma \in\left[\frac{\sqrt{2}}{2}, 1\right]$ such that $\left.u\right|_{\Omega_{\varphi} \cap \partial B^{3}(0, \sigma)} \in W^{1,2}\left(\Omega_{\varphi} \cap \partial B^{3}(0, \sigma), \mathbb{S}^{2}\right)$,

$$
\int_{\Omega_{\varphi} \cap \partial B^{3}(0, \sigma)}|Q u-\overline{\bar{u}}|^{2} d \mathcal{H}^{2}+\int_{\partial\left(B^{3}(0, \sigma) \cap G_{\varphi}\right)}|Q u-\overline{\bar{u}}|^{2} d \mathcal{H}^{1} \leq 8\left[\int_{\Omega_{\varphi} \cap \mathbb{B}}|Q u-\overline{\bar{u}}|^{2} d x+\int_{G_{\varphi}}|Q u-\overline{\bar{u}}|^{2} d \mathcal{H}^{2}\right]
$$

and

$$
\int_{\Omega_{\varphi} \cap \partial B^{3}(0, \sigma)}\left|\nabla_{\tan }(Q u)\right|^{2} d \mathcal{H}^{2} \leq 8 \int_{\Omega_{\varphi} \cap \mathbb{B}}|\nabla(Q u)|^{2} d x .
$$

By Lemma A.3 in the Appendix, there exists a constant $c$ independent of $\varphi$ such that

$$
\begin{aligned}
& \int_{\Omega_{\varphi} \cap \mathbb{B}}|u-\overline{\bar{u}}|^{2} d x \leq c \int_{\Omega_{\varphi} \cap \mathbb{B}}|\nabla u|^{2} d x \\
& \int_{G_{\varphi}}|u-\overline{\bar{u}}|^{2} d \mathcal{H}^{2} \leq c \int_{\Omega_{\varphi} \cap \mathbb{B}}|\nabla u|^{2} d x .
\end{aligned}
$$


Combining inequalities (3.8), (3.9), (3.10), (3.11) and Lemma 3.7 we have

$$
\begin{aligned}
& \left(\int_{\Omega_{\varphi} \cap \partial B^{3}(0, \sigma)}\left|\nabla_{\text {tan }}(Q u)\right|^{2} d \mathcal{H}^{2}\right)\left(\int_{\partial\left(B^{3}(0, \sigma) \cap G_{\varphi}\right)}|Q u-\overline{\bar{u}}|^{2} d \mathcal{H}^{1}+\int_{\Omega_{\varphi} \cap \partial B^{3}(0, \sigma)}|Q u-\overline{\bar{u}}|^{2} d \mathcal{H}^{2}\right) \\
& \leq 64\left(\int_{\Omega_{\varphi} \cap \mathbb{B}}|\nabla(Q u)|^{2} d x\right)\left(\int_{G_{\varphi}}|Q u-\overline{\bar{u}}|^{2} d \mathcal{H}^{2}+\int_{\Omega_{\varphi} \cap \mathbb{B}}|Q u-\overline{\bar{u}}|^{2} d x\right) \\
& \leq 2304\left(\|\varphi\|_{C^{2}}^{2} \mathcal{L}^{3}\left(\Omega_{\varphi} \cap \mathbb{B}\right)+\int_{\Omega_{\varphi} \cap \mathbb{B}}|\nabla u|^{2} d x\right) \times \\
& \left(\int_{\Omega_{\varphi} \cap \mathbb{B}}|Q u-u|^{2}+|u-\overline{\bar{u}}|^{2} d x+\int_{G_{\varphi}}|Q u-u|^{2}+|u-\overline{\bar{u}}|^{2} d \mathcal{H}^{2}\right) \\
& \leq 2304 c\left(\|\varphi\|_{C^{2}}^{2}+\int_{\Omega_{\varphi} \cap \mathbb{B}}|\nabla u|^{2} d x\right) \times \\
& \left(\operatorname{Lip}(\varphi) \mathcal{H}^{2}\left(G_{\varphi}\right)+\operatorname{Lip}(\varphi) \mathcal{L}^{3}\left(\Omega_{\varphi}\right)+2 c \int_{\Omega_{\varphi} \cap \mathbb{B}}|\nabla u|^{2} d x\right) \\
& \leq c\left(\|\varphi\|_{C^{2}}^{2}+\int_{\Omega_{\varphi} \cap \mathbb{B}}|\nabla u|^{2} d x\right)\left(\operatorname{Lip}(\varphi)+\int_{\Omega_{\varphi} \cap \mathbb{B}}|\nabla u|^{2} d x\right)
\end{aligned}
$$

where $c$ has absorbed all constants. Note that as

$$
\mathcal{L}^{3}\left(\Omega_{\varphi} \cap \mathbb{B}\right) \leq \mathcal{L}^{3}(\mathbb{B}) \text { and } \mathcal{H}^{2}\left(G_{\varphi}\right) \leq \int_{\mathbb{B}^{2}} 1+|\nabla \varphi|^{2} d x \leq 2 \mathcal{L}^{2}\left(\mathbb{B}^{2}\right)
$$

the constant $c$ can be chosen to not depend on the domain. Defining $\varepsilon:=a \lambda$, for some $0<a<1$ to be chosen later, and choosing $c_{1}$ such that $c_{1}^{2}<a^{q} \frac{\delta^{2}}{c}$, where $\delta>0$ is the constant from Lemma 3.10, we have

$$
\begin{array}{r}
\left(\int_{\Omega_{\varphi} \cap \partial B^{3}(0, \sigma)}\left|\nabla_{\tan }(Q u)\right|^{2} d \mathcal{H}^{2}\right)\left(\int_{\partial\left(B^{3}(0, \sigma) \cap G_{\varphi}\right)}|Q u-\overline{\bar{u}}|^{2} d \mathcal{H}^{1}+\int_{\Omega_{\varphi} \cap \partial B^{3}(0, \sigma)}|Q u-\overline{\bar{u}}|^{2} d \mathcal{H}^{2}\right) \\
\leq c_{1}^{2} \lambda^{q} c \\
\leq \varepsilon^{q} \delta^{2} .
\end{array}
$$

It now follows that $Q u$ satisfies the small oscillation condition of Lemma 3.10. Therefore, there exists $\omega \in W^{1,2}\left(\Omega_{\varphi} \cap B^{3}(0, \sigma), \mathbb{S}^{2}\right)$ such that $\left.\omega\right|_{\Omega_{\varphi} \cap \partial B^{3}(0, \sigma)}=\left.Q u\right|_{\Omega_{\varphi} \cap \partial B^{3}(0, \sigma)}$ 


$$
\begin{aligned}
& ,\left.\omega\right|_{G_{\varphi} \cap B^{3}(0, \sigma)} \in \tilde{\mathbb{S}}^{1} \text { and } \\
& \begin{aligned}
\int_{\Omega_{\varphi} \cap B^{3}(0, \sigma)}|\nabla \omega|^{2} d x \leq c \varepsilon & \int_{\Omega_{\varphi} \cap \partial B^{3}(0, \sigma)}\left|\nabla_{\tan } Q u\right|^{2} d \mathcal{H}^{2} \\
& +c \varepsilon^{-q}\left[\int_{\Omega_{\varphi} \cap \partial B^{3}(0, \sigma)}|Q u-\overline{\bar{u}}|^{2} d \mathcal{H}^{2}+\int_{\partial\left(\Omega_{\varphi} \cap \partial B^{3}(0, \sigma)\right)}|Q u-\overline{\bar{u}}|^{2} d \mathcal{H}^{1}\right] .
\end{aligned}
\end{aligned}
$$

Using (3.8), (3.9), (3.10), (3.11), (3.12) and Lemma 3.7 we bound

$$
\begin{aligned}
\int_{\Omega_{\varphi} \cap B^{3}(0, \sigma)}|\nabla \omega|^{2} d x \leq & 8 \varepsilon \int_{\Omega_{\varphi} \cap \mathbb{B}}|\nabla Q u|^{2} d x+8 c \varepsilon^{-q}\left[\int_{\Omega_{\varphi} \cap \mathbb{B}}|Q u-\overline{\bar{u}}|^{2} d x+\int_{G_{\varphi}}|Q u-\overline{\bar{u}}|^{2} d \mathcal{H}^{2}\right] \\
\leq & c \varepsilon\left(\|\varphi\|_{C^{2}}^{2}+\int_{\Omega_{\varphi} \cap \mathbb{B}}|\nabla u|^{2} d x\right) \\
& +c \varepsilon^{-q}\left[\operatorname{Lip}(\varphi)^{2}+\int_{\Omega_{\varphi} \cap \mathbb{B}}|u-\overline{\bar{u}}|^{2} d x+\int_{G_{\varphi}}|u-\overline{\bar{u}}|^{2} d \mathcal{H}^{2}\right]
\end{aligned}
$$

where $c$ has absorbed all constants.

We now use the inverse of the matrix $Q(x)$ in order to get a map that belongs to $\mathcal{U}_{\varphi}$. For a given point $x \in \partial \Omega_{\varphi}$ the matrix $[Q(x)]^{-1}$ is given by $Q(x)^{T}$, thus using the symmetry of $Q$ we can write $[Q(x)]^{-1}$ out explicitly as

$$
[Q(x)]^{-1}=\left[\begin{array}{ccc}
Q_{11}(x) & Q_{12}(x) & -Q_{13}(x) \\
Q_{21}(x) & Q_{22}(x) & -Q_{23}(x) \\
-Q_{31}(x) & -Q_{32}(x) & Q_{33}(x)
\end{array}\right]
$$

where $Q_{i j}(x)$ are the entries of the matrix $Q(x)$. By the same calculations as before the map $x \mapsto[Q(x)]^{-1}$ is $C^{1}$ and satisfies the bound

$$
\left|\nabla\left([Q(x)]^{-1} \omega(s)\right)\right|^{2} \leq 18\left(\|\varphi\|_{C^{2}}^{2}+c|\nabla \omega|^{2}\right) .
$$

Moreover, as $\left.\omega\right|_{G_{\varphi} \cap B^{3}(0, \sigma)} \in \tilde{\mathbb{S}}^{1}$ we have $\tilde{w}(x):=[Q(x)]^{-1} \omega(x) \in \mathcal{U}_{\Omega_{\varphi} \cap B^{3}(0, \sigma)}$ and $\left.\tilde{w}(x)\right|_{\Omega_{\varphi} \cap \partial B^{3}(0, \sigma)}=\left.u\right|_{\Omega_{\varphi} \cap \partial B^{3}(0, \sigma)}$. 
Using that $\sigma>\frac{1}{2}$ and the minimality of $u$ we have

$$
\begin{aligned}
& \int_{\Omega_{\varphi} \cap B^{3}\left(0, \frac{1}{2}\right)}|\nabla u|^{2} d x-K_{13} \int_{G_{\varphi} \cap B^{3}\left(0, \frac{1}{2}\right)} u^{\alpha} u^{\beta} \frac{\partial \nu^{\alpha}}{\partial x^{\beta}} d \mathcal{H}^{2} \\
& \leq \int_{\Omega_{\varphi} \cap B^{3}(0, \sigma)}|\nabla u|^{2} d x-K_{13} \int_{G_{\varphi} \cap B^{3}(0, \sigma)} u^{\alpha} u^{\beta} \frac{\partial \nu^{\alpha}}{\partial x^{\beta}} d \mathcal{H}^{2} \\
&+K_{13}\left[\int_{G_{\varphi} \cap B^{3}(0, \sigma)} u^{\alpha} u^{\beta} \frac{\partial \nu^{\alpha}}{\partial x^{\beta}} d \mathcal{H}^{2}-\int_{G_{\varphi} \cap B^{3}\left(0, \frac{1}{2}\right)} u^{\alpha} u^{\beta} \frac{\partial \nu^{\alpha}}{\partial x^{\beta}} d \mathcal{H}^{2}\right] \\
& \leq \int_{\Omega_{\varphi} \cap B^{3}(0, \sigma)}|\nabla \tilde{w}|^{2} d x \\
&+K_{13}\left[\int_{G_{\varphi} \cap B^{3}(0, \sigma)}\left(u^{\alpha} u^{\beta}-\tilde{w}^{\alpha} \tilde{w}^{\beta}\right) \frac{\partial \nu^{\alpha}}{\partial x^{\beta}} d \mathcal{H}^{2}-\int_{G_{\varphi} \cap B^{3}\left(0, \frac{1}{2}\right)} u^{\alpha} u^{\beta} \frac{\partial \nu^{\alpha}}{\partial x^{\beta}} d \mathcal{H}^{2}\right]
\end{aligned}
$$

which implies the inequality

$$
\int_{\Omega_{\varphi} \cap B^{3}\left(0, \frac{1}{2}\right)}|\nabla u|^{2} d x \leq \int_{\Omega_{\varphi} \cap B^{3}(0, \sigma)}|\nabla \tilde{w}|^{2} d x+K_{13}\left[\int_{G_{\varphi} \cap B^{3}(0, \sigma)}\left(u^{\alpha} u^{\beta}-\tilde{w}^{\alpha} \tilde{w}^{\beta}\right) \frac{\partial \nu^{\alpha}}{\partial x^{\beta}} d \mathcal{H}^{2}\right] .
$$

Next we apply (3.14) and (3.13), choose $a<\frac{1}{18 c}$ and substitute $\varepsilon=a \lambda$ to get the bound

$$
\begin{aligned}
\int_{\Omega_{\varphi} \cap B^{3}(0, \sigma)}|\nabla \tilde{w}|^{2} d x \leq & \int_{\Omega_{\varphi} \cap B^{3}(0, \sigma)} 18\left(\|\varphi\|_{C^{2}}^{2}+c|\nabla \omega|^{2}\right) d x \\
\leq & 18\left(c \varepsilon\left(\|\varphi\|_{C^{2}}^{2}+\int_{\Omega_{\varphi} \cap \mathbb{B}}|\nabla u|^{2} d x\right)\right. \\
& \left.+c \varepsilon^{-q}\left[\operatorname{Lip}(\varphi)^{2}+\int_{\Omega_{\varphi}}|u-\overline{\bar{u}}|^{2} d x+\int_{G_{\varphi}}|u-\overline{\bar{u}}|^{2} d \mathcal{H}^{2}\right]\right) \\
& +18\|\varphi\|_{C^{2}}^{2} \mathcal{L}^{3}\left(\Omega_{\varphi}\right) \\
\leq & \lambda\left(\|\varphi\|_{C^{2}}^{2}+\int_{\Omega_{\varphi} \cap \mathbb{B}}|\nabla u|^{2} d x\right) \\
& +\lambda^{-q} c_{2}\left(\operatorname{Lip}(\varphi)^{2}+\int_{\Omega_{\varphi} \cap \mathbb{B}}|u-\overline{\bar{u}}|^{2} d x+\int_{G_{\varphi}}|u-\overline{\bar{u}}|^{2} d \mathcal{H}^{2}\right) \\
& +c_{2}\|\varphi\|_{C^{2}}^{2} .
\end{aligned}
$$


On the other hand, as $u$ and $\tilde{w}$ are both $\mathbb{S}^{2}$ valued, we have

$$
\begin{aligned}
K_{13}\left[\int_{G_{\varphi} \cap B^{3}(0, \sigma)}\left(u^{\alpha} u^{\beta}-\tilde{w}^{\alpha} \tilde{w}^{\beta}\right) \frac{\partial \nu^{\alpha}}{\partial x^{\beta}} d \mathcal{H}^{2}\right] & \leq\left|K_{13}\right| \int_{G_{\varphi} \cap B^{3}(0, \sigma)}\left|u^{\alpha} u^{\beta}-\tilde{w}^{\alpha} \tilde{w}^{\beta}\right|\left|\frac{\partial \nu^{\alpha}}{\partial x^{\beta}}\right| d \mathcal{H}^{2} \\
& \leq c \int_{G_{\varphi} \cap B^{3}(0, \sigma)}\left|\frac{\partial \nu^{\alpha}}{\partial x^{\beta}}\right| d \mathcal{H}^{2} .
\end{aligned}
$$

A straight forward calculation gives

$$
\frac{\partial \nu}{\partial x^{\beta}}=\left[\frac{\left(-\varphi_{x^{1}, x^{\beta}},-\varphi_{x^{2}, x^{\beta}}, 0\right)}{\left(\varphi_{x^{1}}^{2}+\varphi_{x^{2}}^{2}+1\right)^{\frac{1}{2}}}-\frac{\left(\varphi_{x^{1}, x^{\beta}} \varphi_{x^{1}}+\varphi_{x^{2}, x^{\beta}} \varphi_{x^{2}}\right)\left(-\varphi_{x^{1}},-\varphi_{x^{2}}, 1\right)}{\left(\varphi_{x^{1}}^{2}+\varphi_{x^{2}}^{2}+1\right)^{\frac{3}{2}}}\right]
$$

hence

$$
\left|\frac{\partial \nu^{\alpha}}{\partial x^{\beta}}\right| \leq 3\|\varphi\|_{C^{2}}
$$

Recalling that $\mathcal{H}^{2}\left(G_{\varphi}\right)$ is bounded for all $\varphi$ we complete the proof.

Lemma 3.12. [Energy Improvement] There are positive constants $\varepsilon, c$ and $\theta<1$ such that if $\varphi$ is as in (3.5), $u$ is a minimizer of $\mathbb{G}_{\varphi}$ amongst maps in $\mathcal{U}_{\varphi}$ with fixed trace on $H_{\varphi}$ and $\int_{\Omega_{\varphi}}|\nabla u|^{2} d x \leq \varepsilon^{2}$, then

$$
\frac{1}{\theta} \int_{C(0, \theta) \cap \Omega_{\varphi}}|\nabla u|^{2} d x \leq \theta \max \left\{\int_{\Omega_{\varphi}}|\nabla u|^{2} d x, c\left(\operatorname{Lip}(\varphi)+\|\varphi\|_{C^{2}}\right)\right\}
$$

Proof. Suppose, for a contradiction, that for fixed $0<\theta<1$ there are sequences $\left(u_{i}\right),\left(\varphi_{i}\right),\left(\varepsilon_{i}\right)$ such that $u_{i}$ is minimizing in $\Omega_{\varphi_{i}}$ and $\varepsilon_{i}^{2}=\int_{\Omega_{\varphi_{i}}}\left|\nabla u_{i}\right|^{2} d x \rightarrow 0$ as $i \rightarrow \infty$, but

$$
\frac{1}{\theta} \int_{C(0, \theta) \cap \Omega_{\varphi_{i}}}\left|\nabla u_{i}\right|^{2} d x>\theta \max \left\{\int_{\Omega_{\varphi_{i}}}\left|\nabla u_{i}\right|^{2} d x, i\left(\operatorname{Lip}\left(\varphi_{i}\right)+\left\|\varphi_{i}\right\|_{C^{2}}\right)\right\} .
$$

This implies that

$$
\begin{aligned}
\frac{1}{\theta} \int_{C(0, \theta) \cap \Omega_{\varphi_{i}}}\left|\nabla u_{i}\right|^{2} d x & >\theta \varepsilon_{i}^{2} \\
\frac{\operatorname{Lip}\left(\varphi_{i}\right)}{\varepsilon_{i}^{2}} & \rightarrow 0 \\
\frac{\left\|\varphi_{i}\right\|_{C^{2}}}{\varepsilon_{i}^{2}} & \rightarrow 0
\end{aligned}
$$


We now consider the normalised functions

$$
v_{i}=\varepsilon_{i}^{-1}\left(u_{i}-\overline{\bar{u}}_{i}\right) .
$$

Then, by Lemma A.3, the sequence $\left\{\left\|v_{i}\right\|_{W^{1,2}\left(\Omega_{\varphi_{i}}\right)}\right\}_{i=1}^{\infty}$ is a bounded sequence in $\mathbb{R}$. As each $v_{i}$ is defined on a different domain we extend them all to a common domain using the extension Lemma A.1 that can be found in the appendix. Let $\mathcal{C}=\{x \in$ $\mathbb{R}^{3}:\left|\left(x^{1}, x^{2}\right)\right|<1$ and $\left.-1<x^{3}<\frac{1}{2}\right\}$. Then there exists, by Lemma A.1, a constant $c$, that is independent of $i$, and functions $\widehat{v}_{i} \in W^{1,2}(\mathcal{C})$ such that $v_{i}=\left.\widehat{v}_{i}\right|_{\Omega_{\varphi_{i}}}$ and

$$
\left\|\widehat{v}_{i}\right\|_{W^{1,2}(\mathcal{C})} \leq c\left\|v_{i}\right\|_{W^{1,2}\left(\Omega_{\varphi_{i}}\right)}
$$

(note that $\Omega_{\varphi_{i}} \subset \mathcal{C}$ for $i$ sufficiently large and so we assume $\Omega_{\varphi_{i}} \subset \mathcal{C}$ for all $i$ ). As $\widehat{v}_{i}$ is bounded in $W^{1,2}(\mathcal{C})$ there exists $\widehat{v} \in W^{1,2}(\mathcal{C})$ such that $\widehat{v}_{i}$ converges weakly (on a subsequence) to $\widehat{v}$ in $W^{1,2}(\mathcal{C})$. Define $v \in W^{1,2}\left(\Omega_{0}\right)$ as $v:=\left.\widehat{v}\right|_{\Omega_{0}}$.

We claim that the function $v$ is harmonic. In order to see this we first note that as $u_{i}$ are minimizers and $u_{i}(x) \cdot \nu(x)=0$ for $x \in \partial G_{\varphi_{i}}$ we have that $u_{i}$ satisfies

$$
\int_{\Omega_{\varphi_{i}}}\left\langle\nabla u_{i}, \nabla \zeta\right\rangle-\left|\nabla u_{i}\right|^{2} u_{i} \cdot \zeta d x=0
$$

for all $\zeta \in C_{0}^{\infty}\left(\Omega_{\varphi_{i}}\right)$. Let $\zeta \in C^{\infty}\left(\mathbb{R}^{3}\right)$ be such that $\operatorname{spt}(\zeta) \subset \Omega_{0}$. Then for sufficiently large $i$ we have $\operatorname{spt}(\zeta) \subset \Omega_{\varphi_{i}}$. Observing that $\nabla u_{i}=\varepsilon_{i} \nabla v_{i}$, substituting this into (3.19) and dividing by $\varepsilon_{i}$ yields

$$
0=\int_{\Omega_{\varphi_{i}}}\left\langle\nabla v_{i}, \nabla \zeta\right\rangle-\varepsilon_{i}\left|\nabla v_{i}\right|^{2} u_{i} \cdot \zeta d x .
$$

Using the uniform bounds on $\left|\nabla v_{i}\right|^{2}, u_{i}$ and $\zeta$, we see that the second term tends to 0 as $i \rightarrow \infty$. As $\operatorname{spt}(\zeta) \subset \Omega_{0} \subset \mathcal{C}$ and we have that $\operatorname{spt}(\zeta) \subset \Omega_{\varphi_{i}} \subset \mathcal{C}$ for $i$ large enough, we can use the weak convergence of $\widehat{v}_{i}$ to $\widehat{v}$ to get

$$
\begin{aligned}
0 & =\lim _{i \rightarrow \infty} \int_{\Omega_{\varphi_{i}}}\left\langle\nabla v_{i}, \nabla \zeta\right\rangle-\varepsilon_{i}\left|\nabla v_{i}\right|^{2} u_{i} \cdot \zeta d x \\
& =\lim _{i \rightarrow \infty}\left[\int_{\mathcal{C}}\left\langle\nabla v_{i}, \nabla \zeta\right\rangle d x\right]+0 \\
& =\int_{\mathcal{C}}\langle\nabla v, \nabla \zeta\rangle d x \\
& =\int_{\Omega_{0}}\langle\nabla v, \nabla \zeta\rangle d x
\end{aligned}
$$


Therefore $v$ is harmonic in $\Omega_{0}$.

We now examine the behaviour of $v$ on $G_{0}$ and we will show that $v$ is regular up to the boundary. In order to do so we introduce the following subspaces of $\mathbb{S}^{2}$ :

$$
\begin{aligned}
\Sigma_{x}^{i}: & =\left\{y \in \mathbb{S}^{2}: y \cdot \nu\left(x^{1}, x^{2}, \varphi_{i}\left(x^{1}, x^{2}\right)\right)=0\right\} \\
\Sigma_{0}: & =\left\{y \in \mathbb{S}^{2}: y \cdot(0,0,1)=0\right\} .
\end{aligned}
$$

Claim: $v(x) \in \operatorname{Tan}\left(\Sigma_{0}, a\right)$ for $\mathcal{H}^{2}$ almost everywhere $x \in G_{0}$.

Proof of Claim: For each $i$ let $Q_{i}$ be the projection defined by (3.6) (that now depends on $i$ ). Let $\tilde{u}_{i}: \Omega_{0} \rightarrow \mathbb{S}^{2}$ be the functions defined by

$$
\tilde{u}_{i}(x):=u_{i}\left(\tilde{x}_{i}\right), \quad \text { where } \tilde{x}_{i}:=\left(x^{1}, x^{2}, x^{3}+\varphi_{i}\left(x^{1}, x^{2}\right)\right) .
$$

and $\tilde{v}_{i}$ to be the functions

$$
\tilde{v}_{i}=\varepsilon_{i}^{-1}\left(\tilde{u}_{i}-\overline{\bar{u}}_{i}\right) .
$$

Note that for almost every $x \in \Omega_{0}$ we have $\tilde{v}_{i}(x) \rightarrow v(x)$. As $u_{i}(x) \in \Sigma_{x}^{i}$ for almost every $x \in G_{\varphi_{i}}$, we have $Q_{i}\left(\tilde{x}_{i}\right) \cdot \tilde{u}_{i}(x) \in \Sigma_{0}$ for almost every $x \in G_{0}$. Then for almost every $x \in G_{0}$ we have

$$
\operatorname{dist}\left(\overline{\bar{u}}_{i}, \Sigma_{0}\right)^{2} \leq\left|Q_{i}\left(\tilde{x}_{i}\right) \tilde{u}_{i}(x)-\overline{\bar{u}}_{i}\right|^{2} .
$$

Averaging this over $G_{0}$ and using Lemma 3.7, relation (3.17) and the Poincaré inequalities gives

$$
\begin{aligned}
\operatorname{dist}\left(\overline{\bar{u}}_{i}, \Sigma_{0}\right)^{2} & \leq\left[\mathcal{H}^{2}\left(G_{0}\right)\right]^{-1} \int_{G_{0}}\left|Q_{i}(x) \tilde{u}_{i}-\overline{\bar{u}}_{i}\right|^{2} d \mathcal{H}^{2} \\
& \leq 2\left[\mathcal{H}^{2}\left(G_{0}\right)\right]^{-1}\left(\int_{G_{0}}\left|Q_{i}\left(\tilde{x}_{i}\right) \tilde{u}_{i}(x)-\tilde{u}_{i}(x)\right|^{2}+\left|\tilde{u}_{i}-\overline{\bar{u}}_{i}\right|^{2} \mathcal{H}^{2}\right) \\
& \leq c \varepsilon_{i}^{2} .
\end{aligned}
$$

Hence, for $i$ sufficiently large, there is a unique nearest point $a_{i}$ of $\overline{\bar{u}}_{i}$ on $\Sigma_{0}$. As $\left(\overline{\bar{u}}_{i}\right)_{i \in \mathbb{N}}$ is a bounded sequence in $\mathbb{R}^{3}$ it has a subsequence converging to some $a \in \mathbb{R}^{3}$. Also $\varepsilon_{i}^{-1}\left|\overline{\bar{u}}_{i}-a_{i}\right|=\frac{\operatorname{dist}\left(\overline{\bar{u}}_{i}, \Sigma_{0}\right)}{\varepsilon_{i}} \leq \frac{\sqrt{c} \varepsilon_{i}}{\varepsilon_{i}}=\sqrt{c}$, is bounded. Thus on subsequence we have

$$
\lim _{i \rightarrow \infty} \overline{\bar{u}}_{i}=a \in \Sigma_{0} \text { and } \lim _{i \rightarrow} \varepsilon_{i}^{-1}\left(\overline{\bar{u}}_{i}-a_{i}\right)=w \in \mathbb{R}^{3} .
$$

As $\left(\overline{\bar{u}}_{i}-a_{i}\right) \in \operatorname{Nor}\left(\Sigma_{0}, a_{i}\right)$ and $a_{i} \rightarrow a$ we have $w \in \operatorname{Nor}\left(\Sigma_{0}, a\right)$. For almost every $x \in G_{0}, \tilde{v}_{i}(x) \rightarrow v(x)$ as $i \rightarrow \infty$. For such an $x$ and $i$ sufficiently large we have that

$$
\left(x^{1}, x^{2}, x^{3}+\varphi_{i}\left(x^{1}, x^{2}\right)\right) \in G_{\varphi_{i}},
$$


and hence $\tilde{u}_{i}(x) \in \sum_{\tilde{x}_{i}}^{i}$. We compute

$$
\begin{aligned}
\lim _{i \rightarrow \infty} \varepsilon_{i}^{-1}\left(Q_{i}(x) \tilde{u}_{i}(x)-\overline{\bar{u}}_{i}\right) & =\lim _{i \rightarrow \infty}\left(\varepsilon_{i}^{-1}\left(Q_{i}(x) \tilde{u}_{i}(x)-\tilde{u}_{i}(x)\right)+\varepsilon_{i}^{-1}\left(\tilde{u}_{i}(x)-\overline{\bar{u}}_{i}\right)\right) \\
& =\lim _{i \rightarrow \infty} \varepsilon_{i}^{-1}\left(Q_{i}(x) \tilde{u}_{i}(x)-\tilde{u}_{i}(x)\right)+v(x),
\end{aligned}
$$

using Lemma 3.7

$$
\begin{aligned}
\lim _{i \rightarrow \infty} \varepsilon_{i}^{-1}\left|Q_{i}(x) \tilde{u}_{i}(x)-\tilde{u}_{i}(x)\right| & \leq \lim _{i \rightarrow \infty} c \frac{\operatorname{Lip}\left(\varphi_{i}\right)}{\varepsilon_{i}} \\
& =0 .
\end{aligned}
$$

Thus

$$
\lim _{i \rightarrow \infty} \varepsilon_{i}^{-1}\left(Q_{i}(x) \tilde{u}_{i}(x)-\overline{\bar{u}}_{i}\right)=v(x) \text { for almost every } x \in G_{0} .
$$

As $\varepsilon_{i}^{-1}\left(Q_{i}(x) \tilde{u}_{i}(x)-a_{i}\right)$ approaches a vector in $\operatorname{Tan}\left(\Sigma_{0}, a\right)$ and $w \in \operatorname{Nor}\left(\Sigma_{0}, a\right)$, we have

$$
\varepsilon_{i}^{-1}\left(\tilde{u}_{i}(x)-a_{i}\right) \cdot \varepsilon_{i}^{-1}\left(\overline{\bar{u}}_{i}-a_{i}\right) \rightarrow 0 \text { as } i \rightarrow \infty .
$$

Thus

$$
\begin{aligned}
v(x) \cdot(-w) & =\lim _{i \rightarrow} \varepsilon_{i}^{-1}\left(\tilde{u}_{i}(x)-\overline{\bar{u}}_{i}\right) \cdot \varepsilon_{i}^{-1}\left(a_{i}-\overline{\bar{u}}_{i}\right) \\
& =\lim _{i \rightarrow}\left(\varepsilon_{i}^{-1}\left(\tilde{u}_{i}(x)-a_{i}\right) \varepsilon_{i}^{-1}\left(a_{i}-\overline{\bar{u}}_{i}\right)\right) \cdot \varepsilon_{i}^{-1}\left(a_{i}-\overline{\bar{u}}_{i}\right) \\
& =|w|^{2} .
\end{aligned}
$$

By averaging over $G_{0}$ we deduce $|w|^{2}=\overline{\bar{v}}(-w)=0$, hence

$$
\begin{aligned}
v(x) & =v(x)+w \\
& =\lim _{i \rightarrow} \varepsilon_{i}^{-1}\left(Q_{i}(x) \tilde{u}_{i}(x)-\overline{\bar{u}}_{i}\right)+\lim _{i \rightarrow} \varepsilon_{i}^{-1}\left(\overline{\bar{u}}_{i}-a_{i}\right) \\
& =\lim _{i \rightarrow} \varepsilon_{i}^{-1}\left(Q_{i}(x) \tilde{u}_{i}(x)-a_{i}\right) \in \operatorname{Tan}\left(\Sigma_{0}, a\right) .
\end{aligned}
$$

This proves the claim.

Next we decompose $v=v^{\top}+v^{\perp}$, where $v^{\top} \in \operatorname{Tan}\left(\Sigma_{0}, a\right)$ and $v^{\perp} \in \operatorname{Nor}\left(\Sigma_{0}, a\right)$. We deduce that both $v^{\top}$ and $v^{\perp}$ are Harmonic inside $\Omega_{0}$ and that $v^{\perp}$ is regular up to $G_{0}$ because it satisfies the boundary condition

$$
v^{\perp}=0 \text { on } G_{0} .
$$

To verify the regularity of $v^{\top}$ up to $G_{0}$ we show $v^{\top}$ satisfies the Neumann boundary condition

$$
\frac{\partial}{\partial x_{m}} v^{\top}=0 \text { on } G_{0}
$$


in a weak sense, i.e

$$
\int_{\Omega_{0}} \nabla v \cdot \nabla \xi d x=0
$$

for any $\xi \in C^{\infty}\left(\overline{\Omega_{0}}, \operatorname{Tan}\left(\Sigma_{0}, a\right)\right)$ with $\left(\partial \Omega_{0} \backslash G_{0}\right) \cap \operatorname{Spt}(\xi)=\emptyset$. For this purpose choose an open neighbourhood $U$ of $\Sigma_{0}$ in $\mathbb{R}^{3}$ such that every point $y \in U$ has a unique nearest point on $\Sigma_{0}$. For $x \in G_{\varphi_{i}}$ and $y \in U$, define the 1-dimensional subspaces of $\mathbb{R}^{3}$ as

$$
T_{i}(x, y)=\left\{t\left(y \times \nu_{i}(x)\right)+y: t \in \mathbb{R}\right\} .
$$

Then for $x=\left(x^{1}, x^{2}, x^{3}\right) \in \Omega_{\varphi_{i}}$ and $y \in U$, define

$$
T_{i}(x, y):=T_{i}\left(\left(x^{1}, x^{2}, \varphi_{i}\left(x^{1}, x^{2}\right)\right), y\right) .
$$

We then have that $\left\{T_{i}(x, y): x \in \Omega_{\varphi_{i}}, y \in U\right\}$ is a smooth field of 1 dimensional subspaces such that

$$
T_{i}(x, y) \subseteq \operatorname{Tan}\left(\mathbb{S}^{2}, y\right) \text { for } x \in G_{\varphi_{i}}, y \in \mathbb{S}^{2} \cap U
$$

and

$$
T_{i}(x, y)=\operatorname{Tan}\left(\Sigma_{x}^{i}, y\right) \text { for } x \in G_{\varphi_{i}} \text { and } y \in \Sigma_{x}^{i} .
$$

Next, define $\Pi_{i}: \Omega_{\varphi_{i}} \times U \times \mathbb{R}^{3} \rightarrow \mathbb{R}^{3}$ such that for $(x, y, z) \in \Omega_{\varphi_{i}} \times U \times \mathbb{R}^{3}, \Pi_{i}(x, y, z)$ is the orthogonal projection of $z$ onto $T_{i}(x, y)$. Explicitly $\Pi_{i}$ is given by

$$
\Pi_{i}(x, y, z)=\frac{\left[\left(y \times \nu_{i}(x)\right) \otimes\left(y \times \nu_{i}(x)\right)\right] z}{\left|y \times \nu_{i}(x)\right|^{2}}+y .
$$

We have the following bounds on the derivatives of $\Pi_{i}$ :

$$
\left|\frac{\partial \prod_{i}(x, y, z)}{\partial x^{j}}\right|<C \varepsilon_{i}^{2}|z|, \quad\left|\frac{\partial \prod_{i}(x, y, z)}{\partial z^{j}}\right| \leq 1, \quad\left|\frac{\partial \Pi_{i}(x, y, z)}{\partial y^{j}}\right|<C|z|,
$$

the proof these bounds can be found in Lemma B.1 of the appendix.

We are now in a position to show that

$$
\int_{\Omega_{0}} \nabla v \cdot \nabla \xi d x=0 .
$$

We use the cut off function

$$
\lambda_{\delta}(t):= \begin{cases}1 & \text { if } 0 \leq t \leq \frac{\delta}{2} \\ 2-2 \delta^{-1} t & \text { if } \frac{\delta}{2}<t \leq \delta \\ 0 & \text { if } \delta<t\end{cases}
$$


to define

$$
\xi_{i}(x)=\lambda_{\delta_{i}}\left(\operatorname{dist}\left(u_{i}(x), \Sigma_{0}\right)\right) \cdot \Pi_{i}\left(x, u_{i}(x), \xi(x)\right),
$$

where $\delta_{i}$ will be determined later. We have

$$
\left\{\begin{array}{l}
\xi_{i}(x) \in \operatorname{Tan}\left(\mathbb{S}^{2}, u_{i}(x)\right) \text { for } x \in \Omega_{\varphi_{i}} \\
\xi_{i}(x) \in \operatorname{Tan}\left(\Sigma_{x}^{i}, u_{i}(x)\right) \text { for } x \in G_{\varphi_{i}} .
\end{array}\right.
$$

Next, for $x \in \bar{\Omega}_{\varphi_{i}}$ let $u_{i}^{t}(x)$ be the solution of

$$
\left\{\begin{array}{l}
\left(\frac{d}{d t}\right) u_{i}^{t}(x)=\xi_{i}(x), \\
u_{i}^{0}(x)=u_{i}(x)
\end{array}\right.
$$

Then $u_{i}^{t} \in W^{1,2}\left(\Omega_{\varphi_{i}}, \mathbb{S}^{2}\right)$ with $\frac{d}{d t} u_{i}^{t} \in \operatorname{Tan}\left(\Sigma_{x}^{i}, u_{i}(x)\right)$ for $x \in G_{\varphi_{i}}$, hence $u_{i}^{t}(x) \in \Sigma_{x}^{i}$ for $x \in G_{\varphi_{i}}$ and $u_{i}^{t}(x)=u_{i}(x)$ for $x \in \partial \Omega_{\varphi_{i}} \backslash G_{\varphi_{i}}$. The minimality of $u_{i}$ implies that

$$
\begin{aligned}
0 & =\left.\frac{d}{d t}\right|_{t=0} \int_{\Omega_{\varphi_{i}}}\left|\nabla u_{i}^{t}(x)\right|^{2} d x-K_{13} \int_{G_{\varphi_{i}}} u_{i}^{t, \alpha} u_{i}^{t, \beta} \frac{\partial \nu^{\beta}}{\partial x^{\alpha}} d \mathcal{H}^{2} \\
& =2 \int_{\Omega_{\varphi_{i}}} \nabla u_{i} \nabla \xi_{i} d x-K_{13} \int_{G_{\varphi_{i}}} \xi_{i}^{\alpha} u_{i}^{\beta} \frac{\partial \nu^{\beta}}{\partial x^{\alpha}}+\xi_{i}^{\beta} u_{i}^{\alpha} \frac{\partial \nu^{\beta}}{\partial x^{\alpha}} d \mathcal{H}^{2} .
\end{aligned}
$$

Let $A_{i}=\left\{x \in \bar{\Omega}_{\varphi_{i}}:\left|u_{i}(x)-\overline{\bar{u}}_{i}\right|^{2} \geq \frac{\delta_{i}^{2}}{4}\right\}$. We have

$$
\int_{A_{i}} \frac{\delta_{i}^{2}}{4} d x \leq \int_{A_{i}}\left|u_{i}(x)-\overline{\bar{u}}_{i}\right|^{2} d x \leq \int_{\Omega_{\psi_{i}}}\left|u_{i}(x)-\overline{\bar{u}}_{i}\right|^{2} d x \leq c \int_{\Omega_{\psi_{i}}}\left|\nabla u_{i}\right|^{2} d x \leq c \varepsilon_{i}^{2}
$$

and hence

$$
\mathcal{L}^{3}\left(A_{i}\right) \leq 4 c \varepsilon_{i}^{2} \delta_{i}^{-2}
$$

Setting $B_{i}=\Omega_{\varphi_{i}} \backslash A_{i}$ we have

$$
\begin{aligned}
\left|\int_{\Omega_{\varphi_{i}}} \nabla v_{i} \cdot \nabla \xi d x\right|= & \varepsilon_{i}^{-1} \mid \int_{\Omega_{\varphi_{i}}} \nabla u_{i} \cdot \nabla \xi-\nabla u_{i} \cdot \nabla \xi_{i} d x \\
& \quad+\frac{K_{13}}{2} \int_{G_{\varphi_{i}}} \xi_{i}^{\alpha} u_{i}^{\beta} \frac{\partial \nu^{\beta}}{\partial x^{\alpha}}+\xi_{i}^{\beta} u_{i}^{\alpha} \frac{\partial \nu^{\beta}}{\partial x^{\alpha}} d \mathcal{H}^{2} \mid \\
\leq & \underbrace{\varepsilon_{i}^{-1}\left|\int_{A_{i}} \nabla u_{i} \cdot\left(\nabla \xi-\nabla \xi_{i}\right) d x\right|}_{:=I_{i}}+\underbrace{\varepsilon_{i}^{-1}\left|\int_{B_{i}} \nabla u_{i} \cdot\left(\nabla \xi-\nabla \xi_{i}\right) d x\right|}_{:=I I_{i}} \\
& \quad+\varepsilon_{i}^{-1}\left|\frac{K_{13}}{2} \int_{G_{\varphi_{i}}} \xi_{i}^{\alpha} u_{i}^{\beta} \frac{\partial \nu^{\beta}}{\partial x^{\alpha}}+\xi_{i}^{\beta} u_{i}^{\alpha} \frac{\partial \nu^{\beta}}{\partial x^{\alpha}} d \mathcal{H}^{2}\right| \\
\leq & I_{i}+I I_{i}+c \varepsilon_{i}^{-1} \mathcal{H}^{2}\left(G_{\varphi_{i}}\right)\left\|\varphi_{i}\right\|_{C^{2} .}
\end{aligned}
$$


We have that $\varepsilon_{i}^{-1} c \mathcal{H}^{2}\left(G_{\varphi_{i}}\right)\left\|\varphi_{i}\right\|_{C^{2}} \rightarrow 0$ as $i \rightarrow \infty$ and so we must show that $I_{i}, I I_{i} \rightarrow 0$ as $i \rightarrow 0$.

$$
I_{i} \rightarrow 0
$$

Using Hölder we have

$$
\begin{aligned}
I_{i} & \leq \varepsilon_{i}^{-1}\left(\int_{A_{i}}\left|\nabla u_{i}\right|^{2} d x\right)^{\frac{1}{2}}\left(2\left(\int_{A_{i}}|\nabla \xi|^{2} d x+\int_{A_{i}}\left|\nabla \xi_{i}\right|^{2} d x\right)\right)^{\frac{1}{2}} \\
& \leq \varepsilon_{i}^{-1}\left(\varepsilon_{i}^{2}\right)^{\frac{1}{2}}\left(2\left(c \varepsilon_{i}^{2} \delta_{i}^{-2}\right)+2 \int_{A_{i}}\left|\nabla \xi_{i}\right|^{2} d x\right) \quad(\text { as }|\nabla \xi(x)|<c \text { for some } c) .
\end{aligned}
$$

We now focus on $\left|\nabla \xi_{i}\right|^{2}$. Writing $\Pi_{i}(x, y, z)=\left(\Pi_{i}^{1}(x, y, z), \Pi_{i}^{2}(x, y, z), \Pi_{i}^{3}(x, y, z)\right), \quad u_{i}=\left(u_{i}^{1}, u_{i}^{2}, u_{i}^{3}\right) \quad$ and $\xi=\left(\xi^{1}, \xi^{2}, \xi^{3}\right)$ we have

$$
\begin{aligned}
& \left|\frac{\partial \xi_{i}^{k}}{\partial x^{j}}\right|^{2}=\left|\frac{\partial}{\partial x^{j}}\left(\lambda_{\delta_{i}}\left(\operatorname{dist}\left(u_{i}(x), \Sigma_{0}\right)\right) \Pi_{i}^{k}\left(x, u_{i}(x), \xi(x)\right)\right)\right|^{2} \\
& =\mid \lambda_{\delta_{i}}^{\prime}\left(\operatorname{dist}\left(u_{i}(x), \Sigma_{0}\right)\right)\left(\sum_{l=1}^{3} \frac{\partial u_{i}^{l}(x)}{\partial x^{j}} \frac{\partial\left(\operatorname{dist}\left(u_{i}(x), \Sigma_{0}\right)\right)}{\partial x^{l}}\right) \Pi_{i}^{k}\left(x, u_{i}(x), \xi(x)\right) \\
& +\left.\lambda_{\delta_{i}}\left(\operatorname{dist}\left(u_{i}(x), \Sigma_{0}\right)\right) \frac{\partial}{\partial x^{j}}\left(\Pi_{i}^{k}\left(x, u_{i}(x), \xi(x)\right)\right)\right|^{2} \\
& \leq c \delta_{i}^{-1}\left|\sum_{l=1}^{3} \frac{\partial u_{i}^{l}(x)}{\partial x^{j}}\right|^{2}+2 \mid \sum_{l=1}^{3}\left[\frac{\partial \Pi_{i}^{k}\left(x, u_{i}(x), \xi(x)\right.}{\partial x^{j}} \delta_{l j}\right. \\
& \left.+\frac{\partial \Pi_{i}^{k}\left(x, u_{i}(x), \xi(x)\right)}{\partial y^{l}} \frac{\partial u_{i}^{l}(x)}{\partial x^{j}}+\frac{\partial \Pi_{i}^{k}\left(x, u_{i}(x), \xi(x)\right.}{\partial z^{l}} \frac{\partial \xi^{l}(x)}{\partial x^{j}}\right]\left.\right|^{2} \\
& \leq c \delta_{i}^{-1} \sum_{l=1}^{3}\left|\frac{\partial u_{i}^{l}(x)}{\partial x^{j}}\right|^{2}+c \sum_{l=1}^{3}\left|\frac{\partial \Pi_{i}^{k}}{\partial x^{l}}\left(x, u_{i}(x), \xi(x)\right)\right|^{2}+c \sum_{1=l}^{3}\left|\frac{\partial u_{i}^{l}}{\partial x^{j}}(x)\right|^{2}+c \sum_{l=1}^{3}\left|\frac{\partial \xi^{l}(x)}{\partial x^{j}}\right|^{2}
\end{aligned}
$$

where in the last line we have used (3.22). Summing this inequality over $j, k$, using 
the estimates (3.22), (3.23) and the fact that $\int_{\Omega_{\varphi_{i}}}|\nabla u|^{2} d x=\varepsilon_{i}^{2}$ yields

$$
\begin{aligned}
\int_{A_{i}}\left|\nabla \xi_{i}\right|^{2} d x & =\int_{A_{i}} \sum_{i, j=1}^{3}\left|\frac{\partial \xi_{i}^{k}(x)}{\partial x^{j}}\right|^{2} d x \\
\leq & \int_{A_{i}} \sum_{i, j=1}^{3}\left[c \delta_{i}^{-1} \sum_{l=1}^{3}\left|\frac{\partial u_{i}^{l}}{\partial x^{j}}\right|^{2}+c \sum_{l=1}^{3}\left|\frac{\partial \prod_{i}^{k}\left(x, u_{i}(x), \xi(x)\right)}{\partial x_{l}}\right|^{2}\right. \\
& \left.+c \sum_{l=1}^{3}\left|\frac{\partial u_{i}^{l}(x)}{\partial x^{j}}\right|^{2}+c \sum_{l=1}^{3}\left|\frac{\partial \xi^{l}(x)}{\partial x^{j}}\right|^{2}\right] d x \\
& \leq c \delta_{i}^{-1} \varepsilon_{i}^{2}+c \varepsilon_{i}^{2}+c \varepsilon_{i}^{2}+c \varepsilon_{i}^{2} \delta_{i}^{-2} \\
& \leq c \delta_{i}^{-1} \varepsilon_{i}^{2}+4 c \varepsilon_{i}^{2} \delta_{i}^{-2}(c+c)+c \varepsilon_{i}^{2}
\end{aligned}
$$

Therefore, by combining (3.24) and (3.26) we get

$$
I_{i} \leq 2\left(c \varepsilon_{i}^{2} \delta_{i}^{-1}\right)+c \delta_{i}^{-1} \varepsilon_{i}^{2}+4 c \varepsilon_{i}^{2} \delta_{i}^{-2}\left(c \varepsilon_{i}^{2}+c\right)+c \varepsilon_{i}^{2} \rightarrow 0 \text { as } i \rightarrow \infty,
$$

providing $\delta_{i}=\varepsilon_{i}^{\frac{1}{3}} b$, where $b$ is a constant to be chosen later.

We now show $I I_{i} \rightarrow 0$. Recall that for $x \in B_{i}$ we have $\left|u_{i}(x)-\overline{\bar{u}}_{i}\right| \leq \frac{\delta_{i}}{4}$ and so for $x \in B_{i}$

$$
\begin{aligned}
\operatorname{dist}\left(u_{i}(x), \Sigma_{0}\right) & \leq \operatorname{dist}\left(u_{i}(x), \overline{\bar{u}}_{i}\right)+\operatorname{dist}\left(\overline{\overline{u_{i}}}, \Sigma_{0}\right) \\
& \left.\leq \frac{\delta_{i}}{4}+c \varepsilon_{i} \quad \text { (by inequality } 3.20\right) \\
& \leq \frac{\delta_{i}}{2} \quad\left(\text { providing } \delta_{i}=4 c \varepsilon_{i}^{\frac{1}{3}}\right)
\end{aligned}
$$

For $x \in B_{i}$ we have

$$
\begin{aligned}
\xi_{i}(x) & =\lambda_{\delta_{i}}\left(\operatorname{dist}\left(u_{i}(x), \Sigma_{0}\right)\right) \cdot \Pi_{i}\left(x, u_{i}(x), \xi(x)\right) \\
& =\Pi_{i}\left(x, u_{i}(x), \xi(x)\right) .
\end{aligned}
$$

As $\xi \in \operatorname{Tan}\left(\Sigma_{0}, a\right)$ we have $\xi(x)=\Pi_{i}(0, a, \xi(x))$, hence

$$
\begin{aligned}
\varepsilon_{i}^{-1}\left|\int_{B_{i}} \nabla u_{i}\left(\nabla \xi-\nabla \xi_{i}\right) d x\right| & =\varepsilon_{i}^{-1}\left|\int_{B_{i}} \nabla u_{i}\left(\nabla \Pi_{i}(0, a, \xi)-\nabla \Pi_{i}\left(x, u_{i}(x), \xi\right)\right) d x\right| \\
\leq & \underbrace{\varepsilon_{i}^{-1}\left|\int_{B_{i}} \nabla u_{i}\left(\nabla \Pi_{i}(0, a, \xi)-\nabla \Pi_{i}\left(0, \overline{\bar{u}}_{i}, \xi\right)\right) d x\right|}_{:=I I I_{i}} \\
& \underbrace{\varepsilon_{i}^{-1}\left|\int_{B_{i}} \nabla u_{i}\left(\nabla \Pi_{i}\left(x, u_{i}(x), \xi\right)-\nabla \Pi_{i}\left(0, \bar{u}_{i}, \xi\right)\right) d x\right|}_{:=I V_{i}} .
\end{aligned}
$$


We now show that $I I I_{i}$ and $I V_{i}$ both go to zero separately. Using Hölder's inequality and Lemma B.2 from the appendix we estimate

$$
\begin{aligned}
I I I_{i} & \leq \varepsilon_{i}^{-1}\left(\int_{B_{i}}\left|\nabla u_{i}\right|^{2} d x\right)^{\frac{1}{2}}\left(\int_{B_{i}}\left(\nabla \Pi_{i}(0, a, \xi)-\nabla \Pi_{i}\left(0, \overline{\bar{u}}_{i}, \xi\right)\right)^{2} d x\right)^{\frac{1}{2}} \\
& =\left[\int_{B_{i}}\left|\sum_{j, k=1}^{3}\left(\frac{\partial \Pi_{i}^{k}(0, a, \xi)}{\partial x^{j}}-\frac{\partial \Pi_{i}^{k}\left(0, \overline{\bar{u}}_{i}, \xi\right)}{\partial x^{j}}\right)\right|^{2} d x\right]^{\frac{1}{2}} \\
& =\left[\int_{B_{i}}\left|\sum_{j, k=1}^{3}\left(\sum_{l=1}^{3} \frac{\partial \Pi_{i}^{k}}{\partial z_{l}}(0, a, \xi) \frac{\partial \xi^{l}}{\partial x^{j}}-\frac{\partial \Pi_{i}^{k}}{\partial z_{l}}\left(0, \overline{\bar{u}}_{i}, \xi\right) \frac{\partial \xi^{l}}{\partial x^{j}}\right)\right|^{2} d x\right]^{\frac{1}{2}} \\
& \leq\left[\int_{B_{i}} c\left|a-\overline{\bar{u}}_{i}\right| d x\right]^{\frac{1}{2}} \rightarrow 0 \text { as } i \rightarrow \infty .
\end{aligned}
$$

$$
\begin{aligned}
I V_{i} & \rightarrow 0 \\
I V_{i} & =\varepsilon_{i}^{-1}\left|\int_{B_{i}} \nabla u_{i} \cdot\left[\nabla \Pi_{i}\left(x, u_{i}, \xi\right)-\nabla \Pi_{i}\left(0, \overline{\bar{u}}_{i}, \xi\right)\right] d x\right| \\
& \leq[2 \underbrace{\int_{B_{i}}\left|\nabla \Pi_{i}\left(x, u_{i}, \xi\right)-\nabla \Pi_{i}\left(x, \overline{\bar{u}}_{i}, \xi\right)\right|^{2} d x}_{:=V_{i}}+\underbrace{\int_{B_{i}}\left|\nabla \Pi_{i}\left(x, \overline{\bar{u}}_{i}, \xi\right)-\nabla \Pi_{i}\left(0, \overline{\bar{u}}_{i}, \xi\right)\right|^{2} d x}_{:=V I_{i}}]^{\frac{1}{2}} \\
& =\left(2\left(V_{i}+V I_{i}\right)\right)^{\frac{1}{2}}
\end{aligned}
$$

If we can show $V_{i}, V I_{i} \rightarrow 0$ then $I V_{i} \rightarrow 0$ and hence $I I_{i} \rightarrow 0$. Using Lemma B.2 and 
(3.22) we have

$$
\begin{aligned}
V_{i}= & \int_{B_{i}}\left|\nabla \Pi_{i}\left(x, u_{i}, \xi\right)-\nabla \Pi_{i}\left(x, \overline{\bar{u}}_{i}, \xi\right)\right|^{2} d x \\
= & \int_{B_{i}} \sum_{j, k=1}^{3} \mid \sum_{l=1}^{3} \delta_{l j}\left(\frac{\partial \Pi_{i}^{k}}{\partial x_{l}}\left(x, u_{i}, \xi\right)-\frac{\partial \Pi_{i}^{k}}{\partial x_{l}}\left(x, \overline{\bar{u}}_{i}, \xi\right)\right)+\sum_{l=1}^{3} \frac{\partial u_{i}^{l}}{\partial x^{j}} \frac{\partial \Pi_{i}^{k}}{\partial y_{l}}\left(x, u_{i}, \xi\right) \\
& \quad+\left.\sum_{l=1}^{3} \frac{\partial \xi_{l}}{\partial x^{j}}\left(\frac{\partial \Pi_{i}^{k}}{\partial z_{l}}\left(x, u_{i}, \xi\right)-\frac{\partial \Pi_{i}^{k}}{\partial z_{l}}\left(x, \overline{\bar{u}}_{i}, \xi\right)\right)\right|^{2} d x \\
\leq & \int_{B_{i}} c \varepsilon_{i}^{2}+c \varepsilon_{i}^{2}+c\left|u_{i}(x)-\overline{\bar{u}}_{i}\right|^{2} d x+c \int_{\Omega_{\varepsilon_{i}}}\left|\nabla u_{i}\right|^{2} d x \\
\leq & \int_{B_{i}} 2 c \varepsilon_{i}^{2}+c \delta_{i}^{2} d x+c \varepsilon_{i}^{2} \rightarrow 0 .
\end{aligned}
$$

Finally,

$$
\begin{aligned}
V I_{i} & =\int_{B_{i}}\left|\nabla \Pi_{i}\left(x, \overline{\bar{u}}_{i}, \xi\right)-\nabla \Pi_{i}\left(0, \overline{\bar{u}}_{i}, \xi\right)\right|^{2} d x \\
& =\int_{B_{i}} \sum_{j, k=1}^{3}\left|\sum_{l=1}^{3} \delta_{j l} \frac{\partial \Pi_{i}^{k}}{\partial x_{l}}\left(x, \overline{\bar{u}}_{i}, \xi\right)+\sum_{l=1}^{3} \frac{\partial \xi_{l}}{\partial x^{j}}\left(\frac{\partial \Pi_{i}^{k}}{\partial z_{l}}\left(x, \overline{\bar{u}}_{i}, \xi\right)-\frac{\partial \Pi_{i}^{k}}{\partial z_{l}}\left(0, \overline{\bar{u}}_{i}, \xi\right)\right)\right|^{2} .
\end{aligned}
$$

Using Lemma B.3 we have

$$
\left|\frac{\partial \Pi_{i}^{k}}{\partial z_{l}}(x, y, z)-\frac{\partial \Pi_{i}^{k}}{\partial z_{l}}(0, y, z)\right| \leq c\left|\nu_{i}(x)-\nu_{i}(0)\right| \leq c \operatorname{Lip}\left(\varphi_{i}\right) \leq c \varepsilon_{i} .
$$

Therefore

$$
V I_{i} \leq \int_{B_{i}} c \varepsilon_{i}^{2}+c \varepsilon_{i} d x \rightarrow 0 \text { as } i \rightarrow 0,
$$

and thus we have shown

$$
\int_{\Omega_{0}} \nabla v \cdot \nabla \xi d x=0
$$

It now follows that $v^{\perp}$ and $v^{\top}$ extend by even and odd reflections to functions harmonic on $\mathcal{C}$. Thus, since $\overline{\bar{v}}=0$ and $\int_{\Omega_{0}}|\nabla v|^{2} d x \leq 1$, we have

$$
\begin{aligned}
r^{-3} \int_{C(0, r) \cap \Omega_{0}}|v|^{2} d x & \leq c r^{2} \int_{\Omega_{0}}|v|^{2} d x \\
& \leq c r^{2} \int_{\Omega_{0}}|\nabla v|^{2} d x \\
& \leq c r^{2}
\end{aligned}
$$


The first inequality follows from standard linear elliptic theory (similar to the proof of Lemma 2.2 in [5]) and the second follows using Lemma A.3. The second inequality of (3.27) along with the Poincaré inequality and trace theory also implies

$$
r^{-4} \int_{C(0, r) \cap G_{0}}|v|^{2} d \mathcal{H}^{2} \leq r^{-3}\|v\|_{H^{\frac{1}{2}}\left(C(0, r) \cap G_{0}\right)}^{2} \leq c r^{2} .
$$

We now use Vitali convergence Theorem (Theorem A.2) to show that $\left\|v_{i}\right\|_{L^{2}\left(\Omega_{\varphi_{i}}\right)} \rightarrow$ $\|v\|_{L^{2}\left(\Omega_{0}\right)}$. As $\widehat{v}_{i}$ converges weakly in $W^{1,2}(\mathcal{C})$ to $\widehat{v}$, we have that $\widehat{v}_{i}$ converges strongly in $L^{2}(\mathcal{C})$ (on a subsequence) to $\widehat{v}$. Hence we have

$$
\widehat{v}_{i} \mathbb{1}_{\Omega_{\varphi_{i}}}(x) \rightarrow v \mathbb{1}_{\Omega_{0}}(x)
$$

for $\mathcal{L}^{3}$ almost everywhere $x \in \mathcal{C}$, where $\mathbb{1}_{\Omega_{\varphi_{i}}}$ and $\mathbb{1}_{\Omega_{0}}$ are the indicator functions of $\Omega_{\varphi_{i}}$ and $\Omega_{0}$ respectively. Using the Sobolev embedding Theorem we have

$$
\left\|v_{i}\right\|_{L^{3}\left(\Omega_{\varphi_{i}}\right)} \leq\left\|\widehat{v}_{i}\right\|_{L^{3}(\mathcal{C})} \leq C^{\prime}\left\|\widehat{v}_{i}\right\|_{W^{1,2}(\mathcal{C})} \leq C^{\prime} C\left\|v_{i}\right\|_{W^{1,2}\left(\Omega_{\varphi_{i}}\right)} \leq K .
$$

Let $E \subset \mathcal{C}$ be an arbitrary measurable set. Then Hölders inequality and inequality (3.29) yields

$$
\begin{aligned}
\int_{E}\left|\widehat{v}_{i} \mathbb{1}_{\Omega_{\varphi_{i}}}\right|^{2} d x & \leq\left(\int_{E}\left|\widehat{v}_{i} \mathbb{1}_{\Omega_{\varphi_{i}}}\right|^{3} d x\right)^{\frac{2}{3}} \mathcal{L}^{3}(E)^{\frac{1}{3}} \\
& \leq K^{\frac{2}{3}}\left(\mathcal{L}^{3}(E)\right)^{\frac{1}{3}} .
\end{aligned}
$$

Therefore for any $\epsilon>0$ there exists a $\delta>0$ such that $\int_{E}\left|\widehat{v}_{i} \mathbb{1}_{\Omega_{\varphi_{i}}}\right|^{2} d x \leq \epsilon$ for all $i$ and all $E \subset \mathcal{C}$ such that $\mathcal{L}^{3}(E)<\delta$. Hence $\left|v_{i} \mathbb{1}_{\Omega_{\varphi_{i}}}\right|^{2}$ is uniformly integrable. Thus by Vitali convergence Theorem we get

$$
\left.\int_{\mathcal{C}}|| \widehat{v}_{i} \mathbb{1}_{\Omega_{\varphi_{i}}}\right|^{2}-\left|v \mathbb{1}_{\Omega_{0}}\right|^{2} \mid d x \rightarrow 0
$$

Therefore for any $\theta<r<1$ we have

$$
\left.\left|f_{\Omega_{\varphi_{i}} \cap C(0, r)}\right| v_{i}\right|^{2} d x-f_{\Omega_{0} \cap C(0, r)}|v|^{2} d x \mid \leq c \theta^{2} \leq c r^{2}
$$

for sufficiently large $i$. We also have $\left\|v_{i}\right\|_{L^{2}\left(G_{\varphi_{i}}\right)} \rightarrow\|v\|_{L^{2}\left(G_{0}\right)}$, hence for sufficiently large $i$ we have

$$
\left.\left|f_{G_{\varphi_{i}} \cap C(0, r)}\right| v_{i}\right|^{2} d \mathcal{H}^{2}-f_{G_{0} \cap C(0, r)}|v|^{2} d \mathcal{H}^{2} \mid \leq c \theta^{2} \leq c r^{2}
$$


Combining (3.27), (3.28), (3.30) and (3.31) we arrive at

$$
f_{\Omega_{\varphi_{i}} \cap C(0, r)}\left|u_{i}-\overline{\bar{u}}_{i}\right|^{2} d x \leq \varepsilon_{i}^{2}\left(c r^{2}+f_{\Omega_{0} \cap C(0, r)}|v|^{2} d x\right) \leq c \varepsilon_{i}^{2} r^{2}
$$

and similarly

$$
f_{G_{\varphi_{i}} \cap C(0, r)}\left|u_{i}-\overline{\bar{u}}_{i}\right|^{2} d \mathcal{H}^{2} \leq c \varepsilon_{i}^{2} r^{2} .
$$

We now use the Hybrid inequality (Lemma 3.11) and the scaling discussed in section 3.2.1 in order to to contradict our assumption that $\frac{1}{\theta} \int_{C(0, \theta) \cap \Omega}\left|\nabla u_{i}\right|^{2} d x>\theta \varepsilon_{i}^{2}$. For each $i$ we apply the Hybrid inequality to the scaled function $\left(u_{i}\right)_{4 \theta}$ to obtain

$$
\begin{aligned}
& \frac{1}{2 \theta} \int_{C(0, \theta) \cap \Omega_{\varphi_{i}}}\left|\nabla u_{i}\right|^{2} d x \\
& \leq \lambda\left(\left\|\left(\varphi_{i}\right)_{4 \theta}\right\|_{C^{2}}^{2}+\frac{1}{4 \theta} \int_{C(0,4 \theta) \cap \Omega_{\varphi_{i}}}\left|\nabla u_{i}\right|^{2} d x\right) \\
&+\lambda^{-q} c\left(\operatorname{Lip}\left(\left(\varphi_{i}\right)_{4 \theta}\right)^{2}+f_{C(0,4 \theta) \cap \Omega_{\varphi_{i}}}\left|u_{i}-\overline{\bar{u}}_{i}\right|^{2} d x+f_{C(0,4 \theta) \cap G_{\varphi_{i}}}\left|u_{i}-\overline{\bar{u}}_{i}\right|^{2} d \mathcal{H}^{2}\right) \\
&+c\left\|\left(\varphi_{i}\right)_{4 \theta}\right\|_{C^{2}}^{2} \\
& \leq \lambda\left[(4 \theta)^{2}\left\|\varphi_{i}\right\|_{C^{2}}^{2}+\frac{1}{4 \theta} \int_{C(0,4 \theta) \cap \Omega_{\varphi_{i}}}\left|\nabla u_{i}\right|^{2} d x\right] \\
&+\lambda^{-q} c\left((4 \theta)^{2} \operatorname{Lip}\left(\varphi_{i}\right)^{2}+f_{C(0,4 \theta) \cap \Omega_{\varphi_{i}}}\left|u_{i}-\overline{\bar{u}}_{i}\right|^{2} d x+f_{C(0,4 \theta) \cap G_{\varphi_{i}}}\left|u_{i}-\overline{\bar{u}}_{i}\right|^{2} d \mathcal{H}^{2}\right) \\
&+c(4 \theta)^{2}\left\|\varphi_{i}\right\|_{C^{2}} .
\end{aligned}
$$


Iterating this $(k-1)$ more times (where $k$ is an integer to be chosen later) gives

$$
\begin{aligned}
& \frac{1}{2 \theta} \int_{C(0, \theta) \cap \Omega_{\varphi_{i}}}\left|\nabla u_{i}\right|^{2} d x \leq \lambda^{k}\left(4^{k} \theta\right)^{-1} \int_{\Omega_{\varphi_{i}} \cap C\left(0,4^{k} \theta\right)}\left|\nabla u_{i}\right|^{2} d x \\
& +\sum_{j=1}^{k} \lambda^{j}\left(4^{j} \theta\right)^{2}\left\|\varphi_{i}\right\|_{C^{2}}^{2} \\
& +\sum_{j=1}^{k} \lambda^{-q} \lambda^{j-1}\left(\left(4^{j} \theta\right)^{2} \operatorname{Lip}\left(\varphi_{i}\right)^{2}+f_{C\left(0,4^{j} \theta\right) \cap \Omega_{\varphi_{i}}}\left|u_{i}-\overline{\bar{u}}_{i}\right|^{2} d x\right. \\
& \left.+f_{C\left(0,4^{j} \theta\right) \cap G_{\varphi_{i}}}\left|u_{i}-\overline{\bar{u}}_{i}\right|^{2} d \mathcal{H}^{2}\right) \\
& +\sum_{j=1}^{k} \lambda^{j-1}\left(4^{j} \theta\right)^{2}\left\|\varphi_{i}\right\|_{C^{2}}^{2}
\end{aligned}
$$

Let $k$ be an integer such that $4^{k} \theta \leq 1 \leq 4^{k+1} \theta$, then using (3.32) and (3.33) we have for $i$ large enough

$$
\begin{gathered}
\frac{1}{2 \theta} \int_{C(0, \theta) \cap \Omega_{\varphi_{i}}}\left|\nabla u_{i}\right|^{2} d x \leq 4 \lambda^{k} \varepsilon_{i}^{2}+\sum_{j=1}^{k} \theta^{2} \varepsilon_{i}^{2}(16 \lambda)^{j} \\
\quad+\lambda^{-q-1} \varepsilon_{i}^{2} \sum_{j=1}^{k}\left[(16 \lambda)^{j} \theta^{2}+2 c \theta^{2}(16 \lambda)^{j}\right]+\lambda^{-1} \varepsilon_{i}^{2} \theta^{2} \sum_{j=1}^{k}(16 \lambda)^{j} \\
\leq 4 \lambda^{k} \varepsilon_{i}^{2}+\lambda^{-l} c \theta^{2}(1-16 \lambda)^{-1} \varepsilon_{i}^{2}
\end{gathered}
$$

where $l=\max \{q+1,1\}$ and $c$ has absorbed all other constants. Set $\lambda=\theta^{\frac{3}{k}}$, then providing $\theta<\frac{1}{4}$ we have $\lambda^{k}=\theta^{3}<\frac{\theta}{16}$. Next, as $k \rightarrow \infty$ as $\theta \rightarrow 0$, we may fix $\theta<\frac{1}{4}$ sufficiently small such that

$$
16 c \theta<\theta^{\frac{3 l}{k}}
$$

Note that for $\theta$ sufficiently small we have $\lambda<\frac{1}{32}$ which implies $(1-16 \lambda)^{-1} \leq 2$ then

$$
\begin{aligned}
\lambda^{-l} c \theta^{2}(1-16 \lambda)^{-1} & =\theta^{-\frac{3 l}{k}} c \theta^{2}(1-16 \lambda)^{-1} \\
& \leq \frac{c \theta^{2}}{16 c \theta}(1-16 \lambda)^{-1} \\
& \leq \frac{\theta}{8}
\end{aligned}
$$

Therefore

$$
\frac{1}{2 \theta} \int_{C(0, \theta) \cap \Omega_{\varphi_{i}}}\left|\nabla u_{i}\right|^{2} d x<\left(\frac{\theta}{4}+\frac{\theta}{8}\right) \varepsilon_{i}^{2}=\frac{3 \theta}{8} \varepsilon_{i}^{2},
$$


contradicting our choice of $u_{i}, \varphi_{i}$.

Theorem 3.13 (Energy Decay). Suppose $\Omega$ is a $C^{2}$ domain in $\mathbb{R}^{3}$ and take $a \in \partial \Omega$. If $u \in \mathcal{U}$ is a minimizer of $\mathbb{G}$ in $\mathcal{U}$ with $\int_{\Omega \cap C(a, R)}|\nabla u|^{2} d x \leq \varepsilon^{2} R$, then

$$
\int_{\Omega \cap C(a, r)}|\nabla u|^{2} d x \leq r^{2} R^{-1} \theta^{-2} \max \left\{\varepsilon^{2}, c\left(R+R^{2}\right)\right\}
$$

for $0<r \leq R$, a suitable $\theta \in(0,1)$ and $c$ a constant depending on $\Omega$.

Proof. We apply inequality (3.15) to the scaled function $v^{1}(x)=u_{\theta R, a}(x)$ to get that for a $\theta \in(0,1)$ as in Lemma 3.12 we have:

$$
\begin{aligned}
\frac{1}{R \theta} \int_{C(a, R \theta) \cap \Omega}|\nabla u(x)|^{2} d x & =\int_{C(a, 1) \cap \Omega}\left|\nabla u_{R \theta, a}(x)\right|^{2} d x \\
& =\frac{1}{\theta} \int_{C(a, \theta) \cap \Omega}\left|\nabla u_{R, a}(x)\right|^{2} d x \\
& \leq \theta \max \left\{\int_{C(a, 1) \cap \Omega}\left|\nabla u_{R, a}(x)\right|^{2} d x, c\left(\operatorname{Lip}\left(\varphi_{R, a}\right)+\left\|\varphi_{R, a}\right\|_{C^{2}}\right)\right\} \\
& \leq \theta \max \left\{\int_{C(a, 1) \cap \Omega}\left|\nabla u_{R, a}(x)\right|^{2} d x, c\left(R \operatorname{Lip}(\varphi)+R^{2}\|\varphi\|_{C^{2}}\right)\right\} .
\end{aligned}
$$

Then, inductively, for $k \in \mathbb{N}$ we get that

$$
\left(\theta^{k-1} R\right)^{-1} \int_{C\left(a, \theta^{k-1} R\right) \cap \Omega}|\nabla u|^{2} d x \leq \theta^{k-1} \max \left\{\varepsilon^{2}, c\left(R \operatorname{Lip}(\varphi)+R^{2}\|\varphi\|_{C^{2}}\right)\right\}
$$

hence

$$
\begin{aligned}
\left(\theta^{k} R\right)^{-1} \int_{C\left(a, \theta^{k} R\right) \cap \Omega}|\nabla u|^{2} d x & =\frac{1}{\theta} \int_{C(a, \theta) \cap \Omega}\left|\nabla u_{\theta^{k-1} R, a}\right|^{2} d x \\
& \leq \theta \max \left\{\int_{C(a, 1)}\left|\nabla u_{\theta^{k-1} R, a}\right|^{2} d x, c\left(\operatorname{Lip}\left(\varphi_{\theta^{k-1} R, a}\right)+\left\|\varphi_{\theta^{k-1} R, a}\right\|_{C^{2}}\right)\right\} \\
& \leq \theta \max \left\{\left[\theta^{k-1} \max \left\{\varepsilon^{2}, c\left(R \operatorname{Lip}(\varphi)+R^{2}\|\varphi\|_{C^{2}}\right)\right\}\right],\right. \\
& \left.c\left(\left(\theta^{k-1} R\right) \operatorname{Lip}(\varphi)+\left(\theta^{k-1} R\right)^{2}\|\varphi\|_{C^{2}}\right)\right\} \\
& =\theta^{k} \max \left\{\varepsilon^{2}, c\left(R \operatorname{Lip}(\varphi)+R^{2}\|\varphi\|_{C^{2}}\right)\right\} .
\end{aligned}
$$


Hence the inequality

$$
\left(\theta^{k} R\right)^{-1} \int_{C\left(a, \theta^{k} R\right) \cap \Omega}|\nabla u|^{2} d x \leq \theta^{k} \max \left\{\varepsilon^{2}, c\left(R \operatorname{Lip}(\varphi)+R^{2}\|\varphi\|_{C^{2}}\right)\right\}
$$

holds for all $k \in \mathbb{N}$. Next, choose $k$ such that $\theta^{k+1} R \leq r<\theta^{k} R$. Then

$$
\begin{aligned}
r^{-1} \int_{C(a, r) \cap \Omega}|\nabla u|^{2} d x & \leq \theta^{-1}\left(\theta^{k} R\right)^{-1} \int_{C\left(a, \theta^{k} R\right) \cap \Omega}|\nabla u|^{2} d x \\
& \leq \theta^{-1} \theta^{k} \max \left\{\varepsilon^{2}, c\left(R \operatorname{Lip}(\varphi)^{2}+R^{2}\|\varphi\|_{C^{2}}^{2}\right)\right\} \\
& \leq \theta^{-1}\left(\theta^{-1} R^{-1} r\right) \max \left\{\varepsilon^{2}, c\left(R \operatorname{Lip}(\varphi)^{2}+R^{2}\|\varphi\|_{C^{2}}^{2}\right)\right\} \\
& \leq \theta^{-2} R^{-1} r \max \left\{\varepsilon^{2}, c\left(R+R^{2}\right)\right\} .
\end{aligned}
$$

\section{Proof. [of Theorem 1.3]}

We note that we just need to look into partial regularity on the boundary, because in the interior of $\Omega$ the energy behaves like that of the standard harmonic map for which the regularity theory is classical (see [17]). Indeed, let $\bar{n}$ be a minimizer of $\mathbb{G}$ and take an arbitrary open set $\omega$ with $\bar{\omega} \subset \Omega$. Let $n_{\omega}$ be a minimizer of $\int_{\omega}|\nabla n|^{2} d x$ in $W^{1,2}\left(\omega ; \mathbb{S}^{2}\right)$ with boundary condition $\left.\bar{n}\right|_{\omega}$. Then defining

$\tilde{n}(x):=\left\{\begin{array}{ll}n(x) & \text { for } x \in \Omega \backslash \omega \\ n_{\omega}(x) & \text { for } x \in \omega\end{array}\right.$ we have that $\tilde{n}$ is a minimizer of $\mathbb{G}$ and thus all the arguments for harmonic maps can be applied to it in $\omega$.

The main point of our work in this last section has been to obtain the energy decay near the boundary, namely Theorem 3.13 before. For a point where the rescaled energy is small enough the standard Morrey lemma shows that the estimate thus obtained implies Hölder regularity. Standard covering arguments show then the set of singularitiy points, those where the renormalized energy is not small, is of zero one-dimensional Hausorf measure (see for instance [7]).

\section{Appendices}

\section{A Some Lemmas for Partial Regularity}

We prove two lemmas that will be useful in the proof of the partial regularity. They are standard results for a fixed domain but we need them to hold with certain constants independent of the domains. 
The first is an extension lemma and it is standard (see for instance [4] Chapter 4 ), but we reproduce a proof for the readers convenience. Its main point is to show that the extension map can be chosen in a manner that depends only on the upper bound of the Lipschitz constant of the boundary.

Lemma A.1. Let $\varphi: \mathbb{R}^{2} \rightarrow \mathbb{R}$ be a Lipschitz map such that $\varphi(0)=|\nabla \varphi(0)|^{2}=0$ and $\operatorname{Lip}(\varphi) \leq \frac{1}{5}$. Then if $f \in W^{1,2}\left(\Omega_{\varphi}\right)$, there exists $\widehat{f} \in W^{1,2}(\mathcal{C})$ such that $\widehat{f}=f$ on $\Omega_{\varphi}$ and

$$
\|\widehat{f}\|_{W^{1,2}(C(0,1))} \leq c\|f\|_{W^{1,2}\left(\Omega_{\varphi}\right)}
$$

where $c$ is independent of $\varphi, f$ and we denote $\mathcal{C}:=\left\{x \in \mathbb{R}^{3}:\left|\left(x^{1}, x^{2}\right)\right|<1\right.$ and $-1<$ $\left.x^{3}<\frac{1}{2}\right\}$ respectively $\Omega_{\varphi}:=\left\{\left(x^{1}, x^{2}, x^{3}\right) \in C(0,1): x^{3}<\varphi\left(x^{1}, x^{2}\right)\right\}$.

Proof. Fix $\varphi$ and define

$$
U:=\mathcal{C} \backslash \bar{\Omega}_{\varphi}
$$

For simplicity we write $x^{\prime}:=\left(x^{1}, x^{2}\right)$. First suppose that $f \in C^{1}\left(\bar{\Omega}_{\varphi}\right)$ and set

$$
\left\{\begin{array}{l}
\widehat{f}(x)=f(x) \text { if } x \in \overline{\Omega_{\varphi}}, \\
\widehat{f}(x)=f\left(x^{\prime},-x^{3}+2 \varphi\left(x^{\prime}\right)\right) \text { if } x \in \bar{U} .
\end{array}\right.
$$

We note that $f=\widehat{f}$ on $\Omega_{\varphi} \cap \mathcal{C}$ and we claim that

$$
\|\widehat{f}\|_{W^{1,2}(U)} \leq c_{1}\|f\|_{W^{1,2}\left(\Omega_{\varphi}\right)} .
$$

To see this let $\psi \in C_{c}^{1}(U)$ and $\left\{\varphi_{k}\right\}_{k=1}^{\infty}$ be a sequence of $C^{\infty}$ functions such that

$$
\left\{\begin{array}{l}
\varphi_{k} \leq \varphi \\
\varphi_{k} \rightarrow \varphi \text { uniformly } \\
D \varphi_{k} \rightarrow D \varphi \text { almost everywhere } \\
\sup _{k}\left\|D \varphi_{k}\right\|_{L^{\infty}}<\infty
\end{array}\right.
$$

Then for $j=1,2$ we have

$$
\begin{aligned}
\int_{U} \widehat{f} \frac{\partial \psi}{\partial x^{j}} d x & =\int_{U} f\left(x^{\prime}, 2 \varphi\left(x^{\prime}\right)-x^{3}\right) \frac{\partial \psi}{\partial x^{j}} d x \\
& =\lim _{k \rightarrow \infty} \int_{U} f\left(x^{\prime}, 2 \varphi_{k}\left(x^{\prime}\right)-x^{3}\right) \frac{\partial \psi}{\partial x^{j}} d x \\
& =-\lim _{k \rightarrow \infty} \int_{U}\left(\frac{\partial f}{\partial x^{j}}\left(x^{\prime}, 2 \varphi_{k}\left(x^{\prime}\right)-x^{3}\right)+2 \frac{\partial f}{\partial x^{3}}\left(x^{\prime}, 2 \varphi_{k}\left(x^{\prime}\right)-x^{3}\right) \frac{\partial \varphi_{k}}{\partial x^{j}}\left(x^{\prime}\right)\right) \psi d x \\
& =-\int_{U}\left(\frac{\partial f}{\partial x^{j}}\left(x^{\prime}, 2 \varphi\left(x^{\prime}\right)-x^{3}\right)+2 \frac{\partial f}{\partial x^{3}}\left(x^{\prime}, 2 \varphi\left(x^{\prime}\right)-x^{3}\right) \frac{\partial \varphi}{\partial x^{j}}\left(x^{\prime}\right)\right) \psi d x
\end{aligned}
$$


In the same way we can compute

$$
\int_{U} \widehat{f} \frac{\partial \psi}{\partial x^{3}} d x=\int_{U} \frac{\partial f}{\partial x^{3}}\left(x^{\prime}, 2 \varphi\left(x^{\prime}\right)-x^{3}\right) \psi d x .
$$

Then, by a change of variables and using the fact $\operatorname{Lip}(\varphi) \leq \frac{1}{5}$ we get

$$
\int_{U}\left|D f\left(x^{\prime}, 2 \varphi\left(x^{\prime}\right)-x^{3}\right)\right|^{2} d x \leq c_{1} \int_{\Omega_{\varphi}}|D f|^{2} d x
$$

for some appropriate $c_{1}$ that is independent of $f$ and $\varphi$. This proves the claim and moreover also shows that $\|\widehat{f}\|_{W^{1,2}(\mathcal{C})} \leq c_{1}\|f\|_{W^{1,2}\left(\Omega_{\varphi}\right)}$. The result for a general $f \in W^{1,2}\left(\Omega_{\varphi}\right)$ can now be achieved via density.

For the proof of the next lemma we will use Vitali convergence theorem which we state here for the readers convenience

Theorem A.2 (Vitali Convergence Theorem). Let $(X, \mathcal{F}, \mu)$ be a positive measure space. Let $f_{n}: X \rightarrow \mathbb{R}, \forall n \in \mathbb{N}$ and $f: X \rightarrow \mathbb{R}$ be measurable functions such that:

a. $\mu(X)<\infty$

b. $\left\{f_{n}\right\}_{n \in \mathbb{N}}$ is uniformly integrable

c. $f_{n}(x) \rightarrow f(x)$ almost everywhere as $n \rightarrow \infty$

d. $|f(x)|<\infty$ almost everywhere

then the following hold

i. $f \in L^{1}(\mu)$

ii. $\lim _{n \rightarrow \infty} \int_{X}\left|f_{n}-f\right| d \mu=0$

We now use this and lemma A.1 to prove the following Poincaré inequality. Its main point is to check that the constant in the inequality can be chosen uniformly for all the domains we consider.

LemmA A.3. Let $\varphi: \mathbb{R}^{2} \rightarrow \mathbb{R}$ be a Lipschitz map such that $\varphi(0)=|\nabla \varphi(0)|^{2}=0$ and $\operatorname{Lip}(\varphi) \leq \frac{1}{2}$. Then there exists $C>0$, independent of $\varphi$, such that for all $u \in W^{1,2}\left(\Omega_{\varphi}\right)$ we have the Poincaré type inequality

$$
\int_{\Omega_{\varphi}}|u-\overline{\bar{u}}|^{2} d x \leq C \int_{\Omega_{\varphi}}|\nabla u|^{2} d x
$$

where $\overline{\bar{u}}:=\mathcal{H}^{2}\left(G_{\varphi}\right)^{-1} \int_{G_{\varphi}} u d \mathcal{H}^{2}$ 
Proof. We argue by contradiction and assume that for all $i \in \mathbb{N}$ there exist $\varphi_{i}, u_{i} \in$ $W^{1,2}\left(\Omega_{\varphi_{i}}\right)$ such that

$$
\frac{\int_{\Omega_{\varphi_{i}}}\left|\nabla u_{i}\right|^{2} d x}{\int_{\Omega_{\varphi_{i}}}\left|u_{i}-\overline{\bar{u}}_{i}\right|^{2} d x}<\frac{1}{i} .
$$

Define the functions $v_{i}:=\left(u_{i}-\overline{\bar{u}}_{i}\right)\left(\int_{\Omega_{\varphi_{i}}}\left|u_{i}-\overline{\bar{u}}_{i}\right|^{2} d x\right)^{-\frac{1}{2}}$. Then we have

$$
\int_{\Omega_{\varphi_{i}}}\left|\nabla v_{i}\right|^{2} d x \leq \frac{1}{i} \quad \text { and } \quad \int_{\Omega_{\varphi_{i}}}\left|v_{i}\right|^{2} d x=1
$$

As $\left|\nabla \varphi_{i}\right|<\frac{1}{2}$, there exists a subsequence (not relabelled) such that $\varphi_{i} \rightarrow \varphi$ uniformly for some $\varphi$.

By Lemma A.1 there exist functions $\widehat{v}_{i} \in W^{1,2}(\mathcal{C})$ such that $\left\|\widehat{v}_{i}\right\|_{W^{1,2}(\mathcal{C})} \leq c\left\|v_{i}\right\|_{W^{1,2}\left(\Omega_{\varphi_{i}}\right)}$ and by inspecting the proof there exists constant $c_{1}$ such that $\left\|\nabla \widehat{v}_{i}\right\|_{L^{2}(\mathcal{C})} \leq c_{1}\left\|\nabla v_{i}\right\|_{L^{2}\left(\Omega_{\varphi_{i}}\right)}$. It follows that there exists $\widehat{v} \in W^{1,2}(\mathcal{C})$ such that $\widehat{v}_{i}$ converge weakly in $W^{1,2}$ to $\widehat{v}$, but as $\left\|\nabla \widehat{v}_{i}\right\|_{L^{2}(\mathcal{C})} \rightarrow 0$ we have that $\widehat{v} \equiv k$ for some constant $k$. We now investigate the behaviour of $\widehat{v}$ on $G_{\varphi}$ where we recall that $G_{\varphi}:=\partial \Omega_{\varphi} \backslash \partial C(0,1)$. We have

$$
\begin{aligned}
\left|\int_{G_{\varphi_{i}}} v_{i} d \mathcal{H}^{2}-\int_{G_{\varphi}} \widehat{v} d \mathcal{H}^{2}\right| & \leq\left|\int_{G_{\varphi_{i}}}\left(v_{i}-\widehat{v}\right) d \mathcal{H}^{2}\right|+\left|\int_{G_{\varphi_{i}}} \widehat{v} d \mathcal{H}^{2}-\int_{G_{\varphi}} \widehat{v} d \mathcal{H}^{2}\right| \\
& \leq\left\|v_{i}-\widehat{v}\right\|_{H^{\frac{1}{2}-\varepsilon}\left(G_{\varphi_{i}}\right)}+\left|\int_{G_{\varphi_{i}}} \widehat{v} d \mathcal{H}^{2}-\int_{G_{\varphi}} \widehat{v} d \mathcal{H}^{2}\right| \\
& \leq\left\|v_{i}-\widehat{v}\right\|_{H^{1-\varepsilon}\left(\Omega_{\varphi_{i}}\right)}+\left|\int_{G_{\varphi_{i}}} \widehat{v} d \mathcal{H}^{2}-\int_{G_{\varphi}} \widehat{v} d \mathcal{H}^{2}\right| \\
& \leq\left\|\hat{v}_{i}-\widehat{v}\right\|_{H^{1-\varepsilon}(\mathcal{C})}+\left|\int_{G_{\varphi_{i}}} \widehat{v} d \mathcal{H}^{2}-\int_{G_{\varphi}} \widehat{v} d \mathcal{H}^{2}\right|
\end{aligned}
$$

As we have $\left\|\widehat{v}_{i}\right\|_{H^{1}(\mathcal{C})}$ bounded uniformly we have that $\widehat{v}_{i} \rightarrow \widehat{v}$ in $H^{1-\varepsilon}(\mathcal{C})$ and since also $\varphi_{i} \rightarrow \varphi$ uniformly the above tends to 0 . Then

$$
0=\int_{G_{\varphi_{i}}} v_{i} d \mathcal{H}^{2} \rightarrow \int_{G_{\varphi}} \widehat{v} d \mathcal{H}^{2}
$$

which implies that $\widehat{v} \equiv 0$.

However, we claim that

$$
1=\lim _{i \rightarrow \infty} \int_{\Omega_{\varphi_{i}}}\left|v_{i}\right|^{2} d x=\int_{\Omega_{\varphi}}|v|^{2} d x
$$


which would contradict $\widehat{v} \equiv 0$.

To prove (A.3) we use Vitali convergence Theorem. First as $\widehat{v}_{i}$ converges strongly in $L^{2}(\mathcal{C})$ to $\widehat{v}$ we have

$$
\widehat{v}_{i}(x) \mathbb{1}_{\Omega_{\varphi_{i}}}(x) \rightarrow \widehat{v}(x) \mathbb{1}_{\Omega_{\varphi}}(x)
$$

for almost every $x \in \mathcal{C}$.

We now show that the sequence of functions $\left\{\left|\widehat{v}_{i} \mathbb{1}_{\Omega_{\varphi_{i}}}\right|^{2}\right\}$ are uniformly integrable. Note that by the Sobolev inequalities, there exists $c_{2}$ such that $\left\|\widehat{v}_{i}\right\|_{L^{3}(\mathcal{C})} \leq c_{2}\left\|\widehat{v}_{i}\right\|_{W^{1,2}(\mathcal{C})}$ hence as $\widehat{v}_{i}$ is bounded in $W^{1,2}(\mathcal{C})$ it is also a bounded sequence in $L^{3}(\mathcal{C})$, say $\left\|\widehat{v}_{i}\right\|_{L^{3}(\mathcal{C})} \leq c_{3}$. Fix $\varepsilon>0$ we want to show that there exists $\delta>0$ such that $\int_{E}\left|\widehat{v}_{i} \mathbb{1}_{\Omega_{\varphi_{i}}}\right|^{2} d x<\varepsilon$ whenever $E \subset \mathcal{C}$ and $\mathcal{L}^{3}(E)<\delta$. With this in mind for $E \subset \mathcal{C}$ we estimate

$$
\begin{aligned}
\int_{E}\left|\widehat{v}_{i} \mathbb{1}_{\Omega_{\varphi_{i}}}\right|^{2} d x & \leq\left(\int_{E}\left|\widehat{v}_{i} \mathbb{1}_{\Omega_{\varphi_{i}}}\right|^{3} d x\right)^{\frac{2}{3}}\left(\int_{E} d x\right)^{\frac{1}{3}} \\
& \leq\left\|\widehat{v}_{i}\right\|_{L^{3}(\mathcal{C})}^{2} \mathcal{L}^{3}(E)^{\frac{1}{3}} \\
& \leq c_{3} \mathcal{L}^{3}(E)^{\frac{1}{3}}
\end{aligned}
$$

thus by setting $\delta=\varepsilon^{3} / c_{3}$ we have shown $\left\{\left|\widehat{v}_{i} \mathbb{1}_{\Omega_{\varphi_{i}}}\right|^{2}\right\}$ are uniformly integrable. We can now conclude using Vitali convergence Theorem that

$$
\left.\lim _{i \rightarrow \infty} \int_{\mathcal{C}}|| \widehat{v}_{i} \mathbb{1}_{\Omega_{\varphi_{i}}}\right|^{2}-\left|\widehat{v} \mathbb{1}_{\Omega_{\varphi}}\right|^{2} \mid d x=0
$$

which implies (A.3) and completes the proof.

\section{B Some bounds on the Projection (3.21)}

We prove some bounds on the derivatives of the projection $\Pi_{i}: \Omega_{\varphi_{i}} \times U \times \mathbb{R}^{3} \rightarrow \mathbb{R}^{3}$ defined by

$$
\Pi_{i}(x, y, z)=\frac{\left[\left(y \times \nu_{i}(x)\right) \otimes\left(y \times \nu_{i}(x)\right)\right] z}{\left|y \times \nu_{i}(x)\right|^{2}}+y,
$$

where $\Omega_{\varphi_{i}}$ and $U$ are as in the proof of Lemma 3.12.

Lemma B.1. Providing $U$ is sufficiently small then there exists $C>0$ such that for large $i$ the projection $\Pi_{i}$ satisfies the bounds

$$
\left|\frac{\partial \Pi_{i}(x, y, z)}{\partial x^{j}}\right|<C \varepsilon_{i}^{2}|z|, \quad\left|\frac{\partial \Pi_{i}(x, y, z)}{\partial z^{j}}\right| \leq 1, \quad\left|\frac{\partial \Pi_{i}(x, y, z)}{\partial y^{j}}\right|<C|z| .
$$


Proof. Writing $y=\left(y^{1}, y^{2}, y^{3}\right)$ a straight forward calculation yields

$$
\begin{aligned}
& \frac{\partial \prod_{i}}{\partial y^{j}}(x, y, z)= \frac{z \cdot\left(\left(\delta_{1 j}, \delta_{2 j}, \delta_{3 j}\right) \times \nu_{i}(x)\right)\left(y \times \nu_{i}(x)\right)}{\left|y \times \nu_{i}(x)\right|^{2}} \\
&+\frac{z \cdot\left(y \times \nu_{i}(x)\right)\left(\left(\delta_{1 j}, \delta_{2 j}, \delta_{3 j}\right) \times \nu_{i}(x)\right)}{\left|y \times \nu_{i}(x)\right|^{2}} \\
&-\frac{2\left(\left(y \times \nu_{i}(x)\right) \cdot\left(\left(\delta_{1 j}, \delta_{2 j}, \delta_{3 j}\right) \times \nu_{i}(x)\right)\right)\left(z \cdot\left(y \times \nu_{i}(x)\right)\right)\left(y \times \nu_{i}(x)\right)}{\left|y \times \nu_{i}(x)\right|^{4}} \\
&+\left(\delta_{1 j}, \delta_{2 j}, \delta_{3 j}\right) \\
& \frac{\partial \prod_{i}}{\partial z^{j}}(x, y, z)=\frac{\left[\left(y \times \nu_{i}(x)\right) \otimes\left(y \times \nu_{i}(x)\right)\right]\left(\delta_{1 j}, \delta_{2 j}, \delta_{3 j}\right)}{\left|y \times \nu_{i}(x)\right|^{2}} \\
& \frac{\partial \prod_{i}}{\partial x^{j}}(x, y, z)=\frac{z \cdot\left(y \times \frac{\partial \nu_{i}(x)}{\partial x^{j}}\right)\left(y \times \nu_{i}(x)\right)}{\left|y \times \nu_{i}(x)\right|^{2}}+\frac{z \cdot\left(y \times \nu_{i}(x)\right)\left(y \times \frac{\partial \nu_{i}(x)}{\partial x^{j}}\right)}{\left|y \times \nu_{i}(x)\right|^{2}} \\
&-\frac{2\left(\left(y \times \nu_{i}(x)\right) \cdot\left(y \times \frac{\partial \nu_{i}(x)}{\partial x^{j}}\right)\right)\left(z \cdot\left(y \times \nu_{i}(x)\right)\right)\left(y \times \nu_{i}(x)\right)}{\left|y \times \nu_{i}(x)\right|^{4}}
\end{aligned}
$$

where

$$
\frac{\partial \nu}{\partial x^{j}}=\left[\frac{\left(-\varphi_{x^{1} x^{j}},-\varphi_{x^{2} x^{j}}, 0\right)}{\left(\varphi_{x^{1}}^{2}+\varphi_{x^{2}}^{2}+1\right)^{\frac{1}{2}}}-\frac{\left(\varphi_{x^{1} x^{j}} \varphi_{x^{1}}+\varphi_{x^{2} x^{j}} \varphi_{x^{2}}\right)\left(-\varphi_{x^{1}},-\varphi_{x^{2}}, 1\right)}{\left(\varphi_{x^{1}}^{2}+\varphi_{x^{2}}^{2}+1\right)^{\frac{3}{2}}}\right] .
$$

We observe that if $U$ is sufficiently small and $i$ sufficiently large then there exists constants $c_{1}, c_{2}>0$ such that $c_{1}<\left|y \times \nu_{i}(x)\right|<c_{2}$. This, along with the inequality (3.18), gives

$$
\left|\frac{\partial \prod_{i}(x, y, z)}{\partial x^{j}}\right|<C \varepsilon_{i}^{2}|z|, \quad\left|\frac{\partial \prod_{i}(x, y, z)}{\partial z^{j}}\right| \leq 1, \quad\left|\frac{\partial \prod_{i}(x, y, z)}{\partial y^{j}}\right|<C|z| .
$$

We next prove a bound on the difference of two projections onto two different lines.

Lemma B.2. Let $y_{1}, y_{2} \in U, x \in \Omega_{\varphi_{i}}$ and $z \in \mathbb{R}^{3}$. Then we have the bound

$$
\left|\frac{\partial \Pi_{i}}{\partial z^{j}}\left(x, y^{1}, z\right)-\frac{\partial \Pi_{i}}{\partial z^{j}}\left(x, y^{2}, z\right)\right| \leq\left|y^{1}-y^{2}\right| .
$$


Proof. First, by writing $\Lambda_{j}=\left(\delta_{1 j}, \delta_{2 j}, \delta_{3 j}\right)$ we have

$$
\begin{aligned}
\frac{\partial \Pi_{i}}{\partial z^{j}}(x, y, z) & =\frac{\left(y \times \nu_{i}(x)\right) \otimes\left(y \times \nu_{i}(x)\right) \Lambda_{j}}{\left|y \times \nu_{i}(x)\right|^{2}} \\
& =A(y, x) \Lambda_{j},
\end{aligned}
$$

where $A(x, y)=\frac{\left(y \times \nu_{i}(x)\right) \otimes\left(y \times \nu_{i}(x)\right)}{\left|y \times \nu_{i}(x)\right|^{2}} \in \mathbb{M}^{3 \times 3}$. We consider the linear map $h: \mathbb{R}^{3} \rightarrow \mathbb{R}^{3}$

$$
z \mapsto\left(A\left(y^{1}, x\right)-A\left(y^{2}, x\right)\right) z,
$$

and calculate the operator norm of $h$. Note that the map $v \mapsto A(x, y) v$ is the projection of $v$ onto the line spanned by $\left(y \times \nu_{i}(x)\right)$. We assume that the plane spanned by $\left(y^{1} \times \nu_{i}(x)\right)$ and $\left(y^{2} \times \nu_{i}(x)\right)$ to be $\mathbb{R}^{2}$ with

$$
\left(\left(y^{1} \times \nu_{i}(x)\right)=(1,0,0) \text { and }\left(y^{2} \times \nu_{i}(x)\right)=(\cos (\tau), \sin (\tau), 0),\right.
$$

where $\tau$ is the angle between $\left(y^{1} \times \nu_{i}(x)\right)$ and $\left(y^{2} \times \nu_{i}(x)\right)$. Then

$$
A\left(y^{1}, x\right)-A\left(y^{2}, x\right)=\left[\begin{array}{ccc}
1-\cos ^{2}(\tau) & -\cos (\tau) \sin (\tau) & 0 \\
-\cos (\tau) \sin (\tau) & -\sin ^{2}(\tau) & 0 \\
0 & 0 & 0
\end{array}\right]
$$

We can find the operator norm of this by finding the largest eigenvalue. A calculation yields

$$
\|h\|_{o p}=|\sin (\tau)| .
$$

Using elementary geometry we have

$$
\begin{aligned}
\sin (\tau) & \leq \frac{\left|\left(y^{1} \times \nu_{i}(x)\right)-\left(y^{2} \times \nu_{i}(x)\right)\right|}{\left|y^{1} \times \nu_{i}(x)\right|} \\
& \leq\left|y^{1}-y^{2}\right| .
\end{aligned}
$$

Therefore

$$
\left|\left(\frac{\left(y^{1} \times \nu_{i}(x)\right) \otimes\left(y^{1} \times \nu_{i}(x)\right)}{\left|\left(y^{1} \times \nu_{i}(x)\right)\right|^{2}}-\frac{\left(y^{2} \times \nu_{i}(x)\right) \otimes\left(y^{2} \times \nu_{i}(x)\right)}{\left|\left(y^{2} \times \nu_{i}(x)\right)\right|^{2}}\right) \Lambda_{j}\right| \leq\left|\Lambda_{j}\right||\sin (\tau)| \leq\left|y^{1}-y^{2}\right| \text {. }
$$

Using similar reasoning one can also prove the following.

Lemma B.3. There exists $C>0$ such that

$$
\left|\frac{\partial \Pi_{i}^{k}}{\partial z_{l}}(x, y, z)-\frac{\partial \Pi_{i}^{k}}{\partial z_{l}}(0, y, z)\right| \leq C\left|\nu_{i}(x)-\nu_{i}(0)\right|
$$

for all $x \in \Omega_{\varphi_{i}}, y \in U$ and $z \in \mathbb{R}^{3}$. 
Proof. We first calculate that

$$
\begin{aligned}
& \left|\frac{\partial \Pi_{i}^{k}}{\partial z_{l}}(x, y, z)-\frac{\partial \Pi_{i}^{k}}{\partial z_{l}}(0, y, z)\right|= \\
& \quad\left|\frac{\left(y \times \nu_{i}(x)\right) \otimes\left(y \times \nu_{i}(x)\right) \Lambda_{j}}{\left|y \times \nu_{i}(x)\right|^{2}}-\frac{\left(y \times \nu_{i}(0)\right) \otimes\left(y \times \nu_{i}(0)\right) \Lambda_{j}}{\left|y \times \nu_{i}(0)\right|^{2}}\right| .
\end{aligned}
$$

This is the difference between two projections on to two different lines, hence by the same reasoning as in the proof of Lemma B.2 we obtain

$$
\left|\frac{\partial \Pi_{i}^{k}}{\partial z_{l}}(x, y, z)-\frac{\partial \Pi_{i}^{k}}{\partial z_{l}}(0, y, z)\right| \leq c\left|\nu_{i}(x)-\nu_{i}(0)\right|
$$

as required.

\section{Notation}

- $\mathbb{E}[n]=\int_{\Omega} \frac{K}{2}|\nabla n|^{2} d x+K_{13} \int_{\partial \Omega}((n \cdot \nabla) n) \cdot \nu d \sigma$

- $\mathscr{A}:=W^{1,2}\left(\Omega ; \mathbb{S}^{d-1}\right) \cap W^{2,1}\left(\Omega ; \mathbb{S}^{d-1}\right)$

- $\Omega \subset \mathbb{R}^{n}$ a is $C^{2}$ domain (See Theorem 1.1)

- $\nu$ unit-norm exterior normal.

- $\mathcal{T}:=\left\{\gamma \in H^{\frac{1}{2}}\left(\partial \Omega, \mathbb{S}^{2}\right): \gamma(x) \cdot \nu(x)=0\right.$ for almost every $\left.x \in \partial \Omega\right\}$

- $\mathcal{U}:=\left\{u \in W^{1,2}\left(\Omega, \mathbb{S}^{2}\right): \operatorname{Trace}(u) \in \mathcal{T}\right\}$

- $\mathbb{G}[n]:=\int_{\Omega} K \sum_{\alpha, \beta=1}^{d} \frac{\partial n^{\alpha}}{\partial x^{\beta}} \frac{\partial n^{\alpha}}{\partial x^{\beta}}-K_{13} \int_{\partial \Omega} \sum_{\alpha, \beta=1}^{d} \frac{\partial \nu^{\beta}}{\partial x^{\alpha}} n^{\beta} n^{\alpha}$.

- $\left\|\varphi_{r, a}\right\|_{C^{2}}:=\max _{|\alpha|=2} \sup _{x \in \mathbb{R}^{2}}\left|\frac{\partial^{|\alpha|} \varphi_{r, a}(x)}{\partial x^{\alpha}}\right|$

- $C(x, r):=\left\{y \in \mathbb{R}^{3}:\left|\left(y^{1}, y^{2}\right)-\left(x^{1}, x^{2}\right)\right|<r,\left|y^{3}-x^{3}\right|<r\right\}$

- $\Omega_{\varphi}=\left\{\left(x^{1}, x^{2}, x^{3}\right) \in C(0,1): x^{3}<\varphi\left(x^{1}, x^{2}\right)\right\}$

- $\Omega_{0}:=\left\{\left(x^{1}, x^{2}, x^{3}\right) \in C(0,1): x^{3}<0\right\}$

- $G_{\varphi}=\partial \Omega_{\varphi} \backslash \partial C(0,1)$

- $G_{0}=\partial \Omega_{0} \backslash \partial C(0,1)$

- $H_{\varphi}=\partial \Omega_{\varphi} \cap \partial C(0,1)$ 
- $H_{0}=\partial \Omega_{0} \cap \partial C(0,1)$

- $\mathbb{G}_{\varphi}[u]:=\int_{\Omega_{\varphi}}|\nabla u|^{2} d x-K_{13} \int_{G_{\varphi}} u^{\alpha} u^{\beta} \frac{\partial \nu^{\beta}}{\partial x^{\alpha}} d x$.

- $B^{d}(0,1)$ is the ball in $\mathbb{R}^{d}$ centred at 0 with radius 1 .

\section{Acknowledgment.}

The activity of Arghir Zarnescu on this work was partially supported by a grant of the Romanian National Authority for Scientific Research and Innovation, CNCSUEFISCDI, project number PN-II-RU-TE-2014-4-0657; by the Basque Government through the BERC 2014-2017 program; and by the Spanish Ministry of Economy and Competitiveness MINECO: BCAM Severo Ochoa accreditation SEV-2013-0323.

Both authors would like to thank the anonymous referee for the careful reading of the manuscript and a number of helpful remarks that helped to improve the presentation.

\section{References}

[1] Alouges, F., and Ghidaglia, J.-M. Minimizing Oseen-Frank energy for nematic liquid crystals: algorithms and numerical results. In Annales de l'IHP Physique théorique (1997), vol. 66, pp. 411-447.

[2] Bröcker, T., AND JÄNICH, K. Introduction to differential topology. Cambridge University Press, Cambridge-New York, 1982. Translated from the German by C. B. Thomas and M. J. Thomas.

[3] Canevari, G., Segatti, A., and Veneroni, M. Morse's index formula in VMO for compact manifolds with boundary. J. Funct. Anal. 269 (2014), 20433082.

[4] Evans, L. C., And Gariepy, R. F. Measure Theory and Fine Properties of Functions. CRC Press, 1992.

[5] Hardt, R., Kinderlehrer, D., And Lin, F.-H. Existence and partial regularity of static liquid crystal configurations. Communications in Mathematical Physics 105 (1986), 547-570.

[6] Hardt, R., And Lin, F. H. Mappings minimizing the $L^{p}$ norm of the gradient. Communications on Pure and Applied Mathematics XL (1987), 555-588. 
[7] Hardt, R., ANd Lin, F. H. Partially constrained boundary conditions with energy minimizing mappings. Communications on Pure and Applied Mathematics (1989).

[8] Hélein, F., And Wood, J. C. Harmonic maps. In Global Analysis. Elsevier Sci. B. V., 2008, pp. 417-491.

[9] Hirsch, M. W. Differential topology, vol. No. 33 of Graduate Texts in Mathematics. Springer-Verlag, New York-Heidelberg, 1976.

[10] LeE, J. M. Introduction to topological manifolds. Graduate Texts in Mathematics. Springer, New York, 2011.

[11] Mazya, V., and Shaposhnikova, T. On the Brezis and Mironescu conjecture concerning a Gagliardo-Nirenberg inequality for fractional Sobolev norms. $J$. Math. Pures Appl. 81 (2002), 877-884.

[12] Morse, M. Singular points of vector fields under general boundary conditions. Amer. J. Math. 51, 2 (1929), 165-178.

[13] Nezza, E. D., Plaatucci, G., and Valdinoci, E. Hitchhikers's guide to the fractional Sobolev spaces. Bull. Sci. math 136 (2012), 521-573.

[14] Oldano, C., and Barbero, G. An ab initio analysis of the second-order elasticity effect on nematic configurations. Physics Letters A 110, 4 (1985), 213216.

[15] Scheven, C. Variationally harmonic maps with general boundary conditions: boundary regularity. Calc. Var. Partial Differential Equations 25, 4 (2006), 409429.

[16] Schoen, R., And Uhlenbeck, K. A regularity theory for harmonic maps. J. Diff. Geometry 17 (1982), 307-335.

[17] Schoen, R., And Uhlenbeck, K. A regularity theory for harmonic maps. J. Differential Geom. 17, 2 (1982), 307-335.

[18] SpIVAK, M. A comprehensive introduction to differential geometry, vol. I. Publish or Perish, Inc., Wilmington, Del., 1979.

[19] TAHERI, A. Function Spaces and Partial Differential Equations: Volume 2Contemporary Analysis. Oxford University Press, 2015. 
[20] Tartar, L. An introduction to Sobolev spaces and interpolation spaces, vol. 3. Springer Science \& Business Media, 2007.

[21] Virga, E. G. Variational theories for liquid crystals, vol. 8 of Applied Mathematics and Mathematical Computation. Chapman \& Hall, London, 1994. 